\title{
RNA Seq analysis of the Eimeria tenella gametocyte transcriptome reveals clues about the molecular basis for sexual reproduction and oocyst biogenesis
}

Robert A Walker ${ }^{1,2}$, Philippa A Sharman ${ }^{3}$, Catherine M Miller ${ }^{3}$, Christoph Lippuner ${ }^{2,4}$, Michal Okoniewski ${ }^{5}$, Ramon M Eichenberger ${ }^{2}$, Chandra Ramakrishnan ${ }^{2}$, Fabien Brossier ${ }^{6,7^{\wedge}}$, Peter Deplazes $^{2}$, Adrian B Hehl $^{2}$ and Nicholas C Smith ${ }^{1^{*}}$

\begin{abstract}
Background: The protozoan Eimeria tenella is a common parasite of chickens, causing avian coccidiosis, a disease of on-going concern to agricultural industries. The high prevalence of E. tenella can be attributed to the resilient oocyst stage, which is transmitted between hosts in the environment. As in related Coccidia, development of the eimerian oocyst appears to be dependent on completion of the parasite's sexual cycle. RNA Seq transcriptome profiling offers insights into the mechanisms governing the biology of E. tenella sexual stages (gametocytes) and the potential to identify targets for blocking parasite transmission.

Results: Comparisons between the sequenced transcriptomes of E. tenella gametocytes and two asexual developmental stages, merozoites and sporozoites, revealed upregulated gametocyte transcription of 863 genes. Many of these genes code for proteins involved in coccidian sexual biology, such as oocyst wall biosynthesis and fertilisation, and some of these were characterised in more depth. Thus, macrogametocyte-specific expression and localisation was confirmed for two proteins destined for incorporation into the oocyst wall, as well as for a subtilisin protease and an oxidoreductase. Homologues of an oocyst wall protein and oxidoreductase were found in the related coccidian, Toxoplasma gondii, and shown to be macrogametocyte-specific. In addition, a microgametocyte gamete fusion protein, EtHAP2, was discovered.
\end{abstract}

Conclusions: The need for novel vaccine candidates capable of controlling coccidiosis is rising and this panel of gametocyte targets represents an invaluable resource for development of future strategies to interrupt parasite transmission, not just in Eimeria but in other Coccidia, including Toxoplasma, where transmission blocking is a relatively unexplored strategy.

Keywords: Eimeria tenella, RNA Seq, Microgametocyte, Macrogametocyte, Oocyst, Fertilisation, Transmission

\footnotetext{
* Correspondence: nicholas.smith@jcu.edu.au

'Deceased

'Queensland Tropical Health Alliance Research Laboratory, Australian Institute of Tropical Health and Medicine, James Cook University, Cairns Campus, McGregor Road, Smithfield, QLD 4878, Australia

Full list of author information is available at the end of the article
} 


\section{Background}

Eimeria tenella is an obligate intracellular protozoan parasite of the phylum Apicomplexa and one of the main causes of avian coccidiosis. Diarrhoea, anaemia and mortality are the main manifestations of clinical coccidiosis but in sub-clinical infections, ineffective feed conversion due to malabsorption is the most important consequence. Coccidiosis is estimated to cost poultry industries around the world in excess of US $\$ 3$ billion per year $[1,2]$. With the development of drug resistance in Eimeria species threatening the continued use of prophylactic anticoccidials, vaccination remains a desirable long-term strategy for combatting this disease [3].

The lifecycle of E. tenella is, arguably, the least complex of all Coccidia and serves as a model to understand the lifecycles of many other important parasites in this group. Toxoplasma gondii, for instance, has a particularly complex lifecycle and is able to infect a variety of intermediate hosts but reproduces sexually only in felids (reviewed in [4]). In E. tenella, following ingestion of a sporulated oocyst, released sporozoites migrate to the intestinal epithelia and undergo three rounds of asexual reproduction, producing successive generations of merozoites. Thirdgeneration merozoites then differentiate into sexual stages (gametocytes). Each microgametocyte produces approximately 100 motile microgametes, each capable of fertilising a single macrogamete. The ensuing zygote encapsulates itself in a protective wall, becoming an oocyst, and is excreted in the faeces of the definitive host to sporulate in the external environment.

The development of the resilient oocyst wall is a crucial feature of the Coccidia and provides remarkable protection, facilitating its essential transmission between hosts. Moreover, disrupting its formation is the basis for the only subunit vaccine against any apicomplexan disease to reach the marketplace, underscoring the potential for controlling parasitic disease by blocking transmission $[5,6]$. However, the development of additional transmission blocking strategies is hampered by our limited understanding of the molecular mechanisms that govern gametocyte and oocyst development.

The differentiation and development of distinct biological stages in the Apicomplexa are dependent on regulated gene transcription. Accordingly, profiling quantitative changes in gene transcription has proven a useful strategy for identifying important stage-specific genes in asexual stages of $E$. tenella [7-9] and T. gondii [10-13], as well as in gametocyte stages of Plasmodium [14,15]. To date, a global analysis of gene transcription has yet to be performed for coccidian gametocytes due, in part, to difficulties producing sufficient quantities of parasite material for conventional transcriptional analysis. However, recent advances in both the sensitivity and affordability of next-generation transcription profiling techniques (i.e. RNA Seq) have opened the door for a thorough analysis of the E. tenella gametocyte transcriptome.

Described in the present study are results from an RNA Seq analysis of E. tenella gametocytes compared with two asexual stages - sporozoites and merozoites. Many of the upregulated gametocyte transcripts identified encode proteins with known or potential roles in parasite transmission. Two oocyst wall proteins, a subtilisin-like protease and an amine oxidase, all specific to macrogametocytes, are characterised in further biological detail, along with a microgametocyte gamete fusion protein, underscoring their potential as transmission blocking targets.

\section{Results and discussion}

Transcriptome sequencing of $E$. tenella gametocytes, merozoites and sporozoites

The dimorphic gametocyte stages of Eimeria carry out specific biological roles, including fertilisation and oocyst wall assembly, which are required for the formation of an infective (sporulated) oocyst and transmission between hosts. In an effort to better characterise these enigmatic stages, transcriptome sequencing was carried out on $E$. tenella gametocytes, comprising a mixture of both macrogametocytes (female) and microgametocytes (male) (Additional file 1a,b). In parallel, the transcriptomes of two different asexual parasite stages, namely third-generation merozoites (112 h post-infection) and sporozoites (excysted from sporulated oocysts), were also sequenced to allow the identification of upregulated transcripts in gametocytes.

Total RNA was extracted from triplicate biological replicates representing each developmental stage, DNasetreated and quality assessed by automated gel electrophoresis (Additional file 1c). Parasite-specific large ribosomal RNA bands (26S and $18 \mathrm{~S}$ ) were detected in all samples, although trace amounts of contaminating host RNA (28S) were observed in gametocyte samples, an unavoidable feature attributed to the methods used to enrich these stages from infected chickens (see Methods); this was not a strong concern since we performed read mapping to the E. tenella genome scaffold and not de novo assembly. Paired-end sequencing was carried out on all RNA samples using the Illumina HiSeq platform. Over 10 million paired-end reads were produced per sample with the exception of one merozoite replicate, $\mathrm{Mb}$, which failed outright and was excluded from further analyses (Additional file 1d).

Paired-end reads were mapped to all 8,786 exon models (representing unique proteins) predicted in the E. tenella genome scaffold (available on www.toxodb. org). The proportion of reads uniquely mapping to $E$. tenella exon models was lower in gametocyte RNA Seq libraries than in merozoites or sporozoites and can be explained by the higher proportion of contaminating host RNA in the gametocyte samples (Additional file 1c). 
Nevertheless, the extraordinary depth of sequencing achieved through Illumina HiSeq - including a total of 79.3 million paired-end reads mapped uniquely to $E$. tenella exon models across the three gametocyte libraries - provided a solid foundation for quantitative assessment of gene transcript levels. A table listing the total number of paired reads mapped to each of the 8,786 predicted exon models in each of the nine RNA Seq libraries is provided (Additional file 2).

\section{Identification of upregulated gametocyte transcripts through analysis of differential expression}

The transcription of genes encoding proteins with important roles in E. tenella gametocyte biology, including oocyst wall biosynthesis [16], protein glycosylation [17] and proteolytic cleavage of oocyst wall proteins [18], is typically restricted to gametocytes and zygotes (early oocysts). In order to identify other upregulated gametocyte transcripts, RNA Seq mapping was used to generate quantitative differential expression (DE) profiles of individual genes between developmental stages of $E$. tenella.

However, prior to this DE analysis, the global transcription profiles of each of the eight RNA Seq libraries were compared in a pair-wise manner using hierarchical clustering (described in Methods). Heat-mapping of the Pearson correlation coefficient scores, confirmed that transcription profiles were most similar between replicates of the same biological stage (Figure 1a). The secondary clustering of gametocyte and merozoite replicates is unsurprising given that third-generation merozoites differentiate into gametocytes. Furthermore, this secondary clustering is also caused by the presence of contaminating a
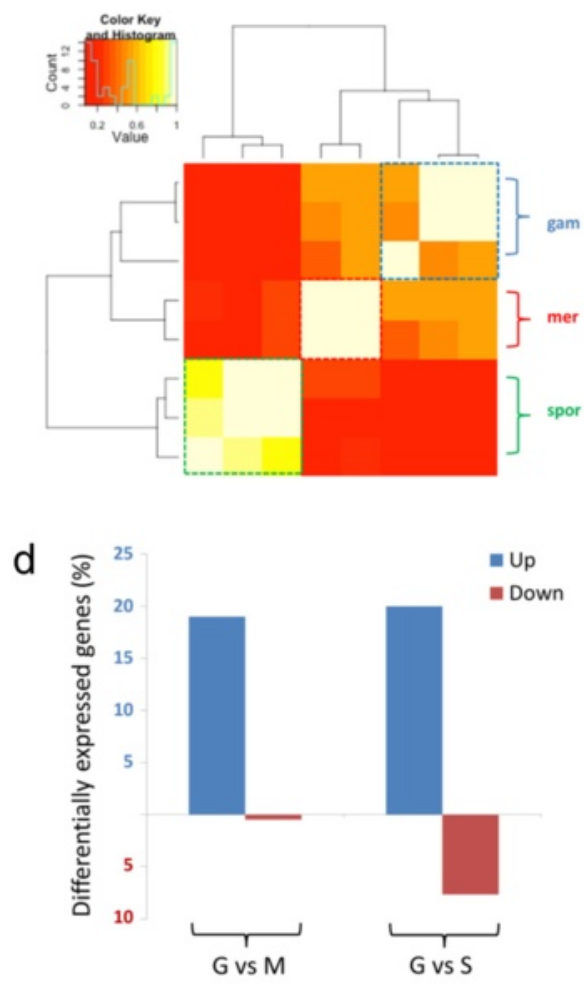

b

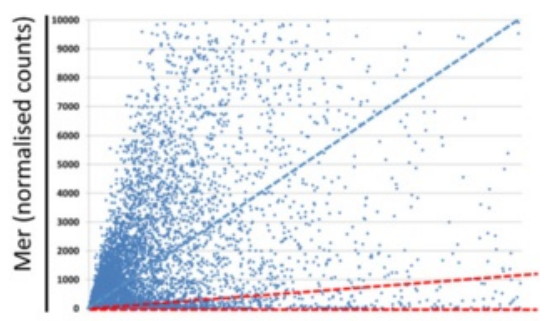

C

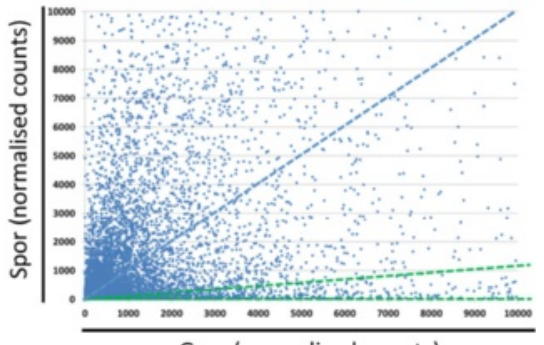

Gam (normalised counts)

e

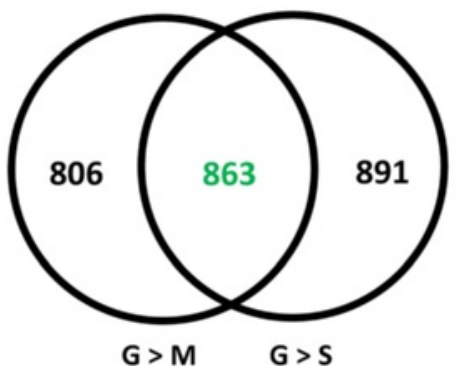

Figure 1 Identification of upregulated gametocyte transcripts using differential expression analysis. (a) The eight RNA Seq libraries were subjected to pair-wise hierarchical clustering between the vectors of read counts for all pairs of samples. Heat-mapping of Pearson's correlation coefficient scores reveal similarities between the transcriptome profiles of the different samples, with white (Pearson $R=1.0$ ) being the most similar and red (Pearson $\mathrm{R}=0.0$ ) being the least similar. Adjacent dendograms reveal clustering of the biological replicates within the same developmental stages, including gametocytes (gam), merozoites (mer) and sporozoites (spor). (b, c) Normalised read counts of individual genes calculated for gametocytes have been plotted against those for merozoites (b) and sporozoites (c). Gene transcripts calculated to be upregulated in gametocytes compared with merozoites or sporozoites reside between dotted red or green dotted-lines, respectively. The dotted blue lines indicate equal levels of transcript abundance between samples. For the sake of clarity, the axis limits have been set to a maximum normalised count of 10,000, inevitably excluding some highly expressed gene transcripts. (d) Summary of the DE analysis of gametocytes compared with merozoites (G vs M) or sporozoites (G vs S) showing the percentage of genes upregulated (blue) or downregulated (red) in gametocytes, of all predicted E. tenella genes. (e) Venn diagram revealing the overlap of genes whose transcript levels were upregulated in gametocytes compared with either merozoites (left, G > M) or sporozoites (right, G > S). A total of 863 upregulated gametocyte transcripts genes were identified in this overlapping region. 
merozoite stages co-purified in gametocyte samples, an unavoidable bi-product of the asynchronicity of $E$. tenella infections at this later time point (144 h post-infection).

Next, normalised read counts were generated for each of the three different biological stages using the DESeq algorithm [19], thereby allowing a comparison of gene transcript levels between different samples. A table listing the normalised counts calculated for each gene in the three biological stages is provided (Additional file 3). A plot of normalised counts for individual gene transcripts in gametocytes against those in merozoites (Figure 1b) or sporozoites (Figure 1c) gives an overview of global DE of gene transcription. Accordingly, genes transcribed specifically in gametocytes plot along or near the $\mathrm{x}$-axis, or along the $y$-axis for either merozoites or sporozoites, while genes showing no DE plot along the dotted blue line (note, the presence of merozoites in the gametocyte samples results in a shift in the plotting of merozoite-specific genes away from the $y$-axis). These pairwise comparisons demonstrate that the transcripts of a large population of genes are detected specifically in gametocytes, mirroring the distinct processes utilised in these sexual stages.

For the purposes of defining stage-specific expression, genes were defined as DE if the log2 fold-change calculated from normalised counts between two stages was equal to or greater than three (or a fold-change $\geq$ eight). The population of upregulated gametocyte transcripts identified using this threshold has been highlighted in the pairwise comparisons with merozoites and sporozoites (dotted red and green lines, respectively - Figure 1b,c). The $\log 2$ fold-changes calculated for all genes in DESeq pairwise comparisons between (i) gametocytes and merozoites or (ii) gametocytes and sporozoites are provided (Additional file 3) along with an adjusted p-value representing false discovery rates. Excluding genes with $\mathrm{DE}$ false discovery rates of $>0.05,1,669$ gene transcripts (19.0\% of all predicted $E$. tenella genes) are upregulated in gametocytes compared to merozoites and 1,754 (20.0\%) in gametocytes compared to sporozoites (Figure 1d). In contrast, fewer genes were calculated with higher transcript levels in merozoites $(44,0.5 \%)$ or sporozoites (675, 7.7\%) compared to gametocytes. The identification of 44 merozoite-specific genes is almost certainly a gross underestimate since the co-purification of merozoites in the gametocyte sample (roughly estimated by light microscopy as $\sim 10 \%$ of the sample) clearly limits the identification of genes with elevated transcript levels in merozoites. However, in the scope of our study, the identification of genes with elevated transcript levels in gametocytes is highly stringent. In total, 863 upregulated gametocyte transcripts were identified (Figure 1e), representing 9.8\% of all predicted $E$. tenella genes. The gene identification, putative biological function and transcript abundance (expressed as FPKM, described below and in the Methods) for each of these genes, in each developmental stage, are provided (Additional file 4).

\section{Highly abundant upregulated gametocyte transcripts}

Within the subset of 863 upregulated gametocyte transcripts identified in E. tenella, the level of transcript abundance for each gene in gametocytes, expressed as FPKM (Fragments Per Kilobase of exon model per Million mapped reads) varies by many orders of magnitude from 27,715.26 (ETH_00019840, hypothetical protein) down to 0.89 (ETH_00021185, hypothetical protein) (Additional file 4). A majority (60.4\%) of all 863 upregulated gametocyte transcripts code for hypothetical proteins (Figure 2), an unsurprising observation given the limited understanding of coccidian sexual biology. However, genes coding for proteins with putative roles in glycosylation, protease activity, redox activity and fatty acid metabolism and as components of microgametes, surface and the oocyst wall, are highlighted in Figure 2 and are described in further detail below. Other putative functions highlighted but not discussed in detail include: (1) cytoskeleton/ transport, processes which may overlap with microgamete flagellar functions; and (2) DNA/RNA binding, which may have unspecified roles in gametocyte-specific gene regulation. The miscellaneous category includes proteins with diverse functions, such as kinase activity, calcium binding, metabolism, etc., whose roles in coccidian gametocyte biology remain unclear and require detailed further study before any meaningful discussion of their functional significance can be attempted.

The two genes with the highest transcript abundance in gametocytes, ETH_00019840 and ETH_00019845, are tandemly located in the E. tenella genome (in Supercontig_2) and code for proteins that share almost 100\% sequence identity (apart from a 44 amino acid insertion within ETH_00019845) (Additional file 5). Both proteins contain a predicted signal peptide and either four (ETH_00019840) or five (ETH_00019845) transmembrane domains. The third and fourth most abundant upregulated gametocyte transcripts, ETH_00019835 and ETH_00032470, code for proteins with predicted signal peptides and 100\% sequence identity over the first 98 amino acids of the $\mathrm{N}$-terminal (Additional file 6). Efforts to characterise the function of these four proteins is on-going.

As expected, genes coding for previously characterised gametocyte antigens and oocyst wall proteins, including EtGAM22, EtGAM56, EtGAM230 and EtGAM59, are among the most highly transcribed gametocyte genes; these are described in greater detail further on. Another highly transcribed gametocyte gene is ETH_00034390, which codes for a macrophage migration inhibitory factor, EtMIF, originally described in E. tenella merozoites [20]. Interestingly, this protein has been subsequently localised to wall-forming bodies of macrogametes and appears to 


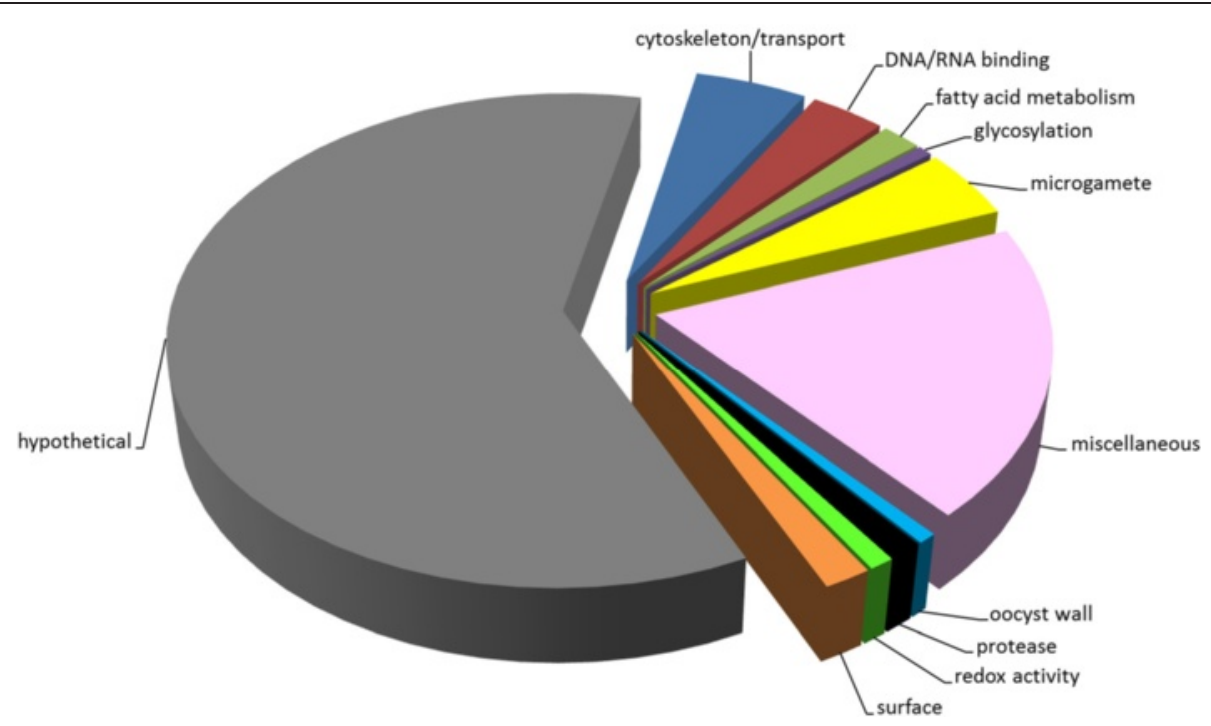

Figure 2 Biological functions of proteins coded by upregulated gametocyte transcripts. The biological function of proteins coded by each of the 863 upregulated gametocyte transcripts was assigned manually based on ToxoDB, Blast2Go or annotations published previously (See Additional file 4). The pie chart represents the proportion of upregulated gametocyte transcripts characterised by these different biological functions.

play a role in modulating the host immune system [21].

\section{Highly abundant transcripts code for novel macrogamete-specific oocyst wall proteins}

Oocyst wall and surface proteins are attractive transmission blocking targets [22-24]. CoxAbic ${ }^{\circ}$, the only commerciallyavailable subunit vaccine against any apicomplexan, comprises macrogamete-specific proteins, GAM56 and GAM82, both of which are destined for incorporation into the oocyst wall [5]. Gene transcripts coding for EtGAM56 and other oocyst wall proteins (EtGAM22, EtGAM59, and EtGAM230) are confirmed here to be upregulated in gametocytes (Table 1). The fifth most abundant upregulated gametocyte transcript detected here, etgam 22, is a multi-copy gene that codes for a histidine- and proline-rich protein [25], while etgam56 and etgam59 code for tyrosine-rich proteins $[16,25]$. Like GAM56 and GAM82, EtGAM22 is a component of the wall forming bodies of macrogametes and is incorporated into the developing oocyst wall $[24,25]$. ETH_00012470 codes for a protein that shows homology to the cysteine-rich oocyst wall proteins, TgOWP6 [26] and CpOWP6 [27] and has been named 'EtOWP6'. This family of proteins is expressed in wall forming bodies of macrogametes and the oocyst walls of $T$. gondii and Cryptosporidium parvum [26,28]. Importantly, EtOWP6 shares the domain architecture described for both TgOWP6 and CpCOWP6 (Additional file 7), specifically the conservation of cysteine-rich Type I repeats [27], supporting its role as a structural component of the oocyst wall. Quantitative RT-PCR confirmed the presence etowp6 transcript in E. tenella gametocytes but also in unsporulated oocysts (Figure 3a). Western blot using anti-EtOWP6 polyclonal antibody detected protein only in gametocyte stages (i.e. not in either oocyst stages), with three bands of 48,45 and $41 \mathrm{kDa}$ (the predicted size of EtOWP6 is $58.5 \mathrm{kDa}$ ) (Figure 3b). It is unclear whether these bands represent different conformations of the one EtOWP6 protein or represent different cross-reactive targets. Immunolocalisation studies of E. tenella-infected chicken intestine using this antibody revealed reactivity against wall-forming bodies type I of macrogametes, with GAM56 antibody used as a counterstain for wall-forming bodies type II (WFBII) (Figure 3c). Surprisingly, EtOWP6 could not be detected in oocyst samples by either Western blot (Figure 3b) or immunolocalisation on both broken and unbroken oocysts (data not shown), despite being detected in proteomic analyses of $E$. tenella oocyst walls [29]. One explanation may be that cross-linking of the cysteine-rich protein during oocyst wall assembly drastically reduced the affinity of this particular antibody; although the reduction and alkylation performed prior to Western blot analyses should have been sufficient for breaking disulphide bonds.

An additional gene, ETH_00018895, codes for a protein that was also detected by mass spectrometry in purified oocyst walls of E. tenella [29] and is hereto referred to as EtHOWP1, for E. tenella Hypothetical Oocyst Wall Protein 1. Unlike etowp6, the ethowp1 transcript was detected exclusively in gametocyte stages of $E$. tenella (Figure 3d). However, Western blot analysis using 
Table 1 Upregulated gametocyte transcripts coding for oocyst wall and surface proteins

\begin{tabular}{|c|c|c|c|}
\hline Gene ID & FPKM & Annotation & Function \\
\hline ETH_00035480 & 8555.22 & EtGAM22 & oocyst wall \\
\hline ETH_00007320 & 2961.32 & EtGAM56 & oocyst wall \\
\hline ETH_00018895 & 2836.86 & EtHOWP1 & oocyst wall \\
\hline ETH_00012470 & 1173.37 & EtOWP6 & oocyst wall \\
\hline ETH_00016615 & 844.43 & EtGAM230 & oocyst wall \\
\hline ETH_00007315 & 544.33 & EtGAM59 & oocyst wall \\
\hline ETH_00022305 & 2917.01 & surface antigen 7 & surface \\
\hline ETH_00024330 & 369.46 & surface antigen 10 & surface \\
\hline ETH_00026205 & 311.85 & PAN domain-containing protein & surface \\
\hline ETH_00034935 & 270.05 & surface antigen 10 & surface \\
\hline ETH_00030195 & 263.42 & fasciclin domain-containing protein & surface \\
\hline ETH_00022280 & 186.18 & surface antigen 7 & surface \\
\hline ETH_00038085 & 175.61 & PAN domain-containing protein & surface \\
\hline ETH_00012815 & 172.72 & PAN domain-containing protein & surface \\
\hline ETH_00027460 & 124.33 & PAN domain-containing protein (EC 3.4.21.27), related & surface \\
\hline ETH_00017025 & 116.54 & plasma-membrane $\mathrm{H}+-$ ATPase & surface \\
\hline ETH_00022855 & 58.90 & plasma membrane calcium-transporting atpase 3-like & surface \\
\hline ETH_00023630 & 44.53 & cell surface glycoprotein 1 & surface \\
\hline ETH_00016215 & 17.17 & outer membrane protein & surface \\
\hline ETH_00023605 & 7.77 & PAN domain-containing protein & surface \\
\hline ETH_00010765 & 4.13 & surface antigen 2 & surface \\
\hline
\end{tabular}

Upregulated gametocyte transcripts coding for known or putative oocyst wall or surface proteins are listed along with their transcript abundance (FPKM), annotation and biological function.

anti-EtHOWP1 polyclonal antibody revealed a doublet band of approximately $40 \mathrm{kDa}$ in gametocytes, unsporulated oocysts and sporulated oocysts, with additional lower molecular weight bands of $30 \mathrm{kDa}$ and $23 \mathrm{kDa}$ in unsporulated and sporulated oocyst samples (Figure 3e). This banding pattern is reminiscent of the proteolytic processing of tyrosine-rich proteins, GAM56 and GAM82, prior to oocyst wall integration [16]; although, in the case of EtHOWP1, the larger $40 \mathrm{kDa}$ protein band persists in oocyst samples. In contrast to EtHOWP1 protein levels, ethowp1 transcript was detected only in gametocyte stages, a feature observed previously with etgam56 [16]. For EtGAM56, the discrepancy between the gene transcript and protein levels is explained by the stockpiling of EtGAM56 protein in WFBII prior to oocyst wall assembly and the high stability of these proteins in the mature oocyst wall structure [16,30]. A similar observation was made for EtHOWP1, which co-localised with EtGAM56 to WFBII (Figure 3f), organelles characterised by their doughnut-like appearance (Figure 3g). WFBII migrate to the periphery of macrogametes and form the inner layer of the developing oocyst wall [31]. Accordingly, when intact and broken oocysts were probed with EtHOWP1 antisera, the protein was detected exclusively in the walls of broken oocysts since the inner layer of the oocyst wall is not accessible in intact oocysts (Figure 3h). In summary, EtHOWP1 is a bona fide oocyst wall protein that, looking forward, should be considered as an attractive vaccine candidate.

Also included in Table 1 are genes that code for predicted surface proteins of $E$. tenella. Of particular interest are ETH_00022305, ETH_00024330, ETH_00034935, ETH_00022280 and ETH_00010765, whose cognate proteins share homology with an immunogenic sporozoite surface antigen (SAG) or TA4 [22]. Another subset of predicted surface proteins are those containing PAN domains (ETH_00026205, ETH_00038085, ETH_00012815, ETH_00027460 and ETH_00023605), which are typically found in proteins involved in adhesion, including in microneme proteins (MICs) of E. tenella [32]. The role of these putative surface proteins in gametocyte biology remains to be investigated.

\section{Identification of macrogamete-specific subtilisins and oxidoreductases with putative roles in oocyst wall biosynthesis}

While abundant oocyst wall proteins represent the structural building blocks for the coccidian oocyst wall, many of 


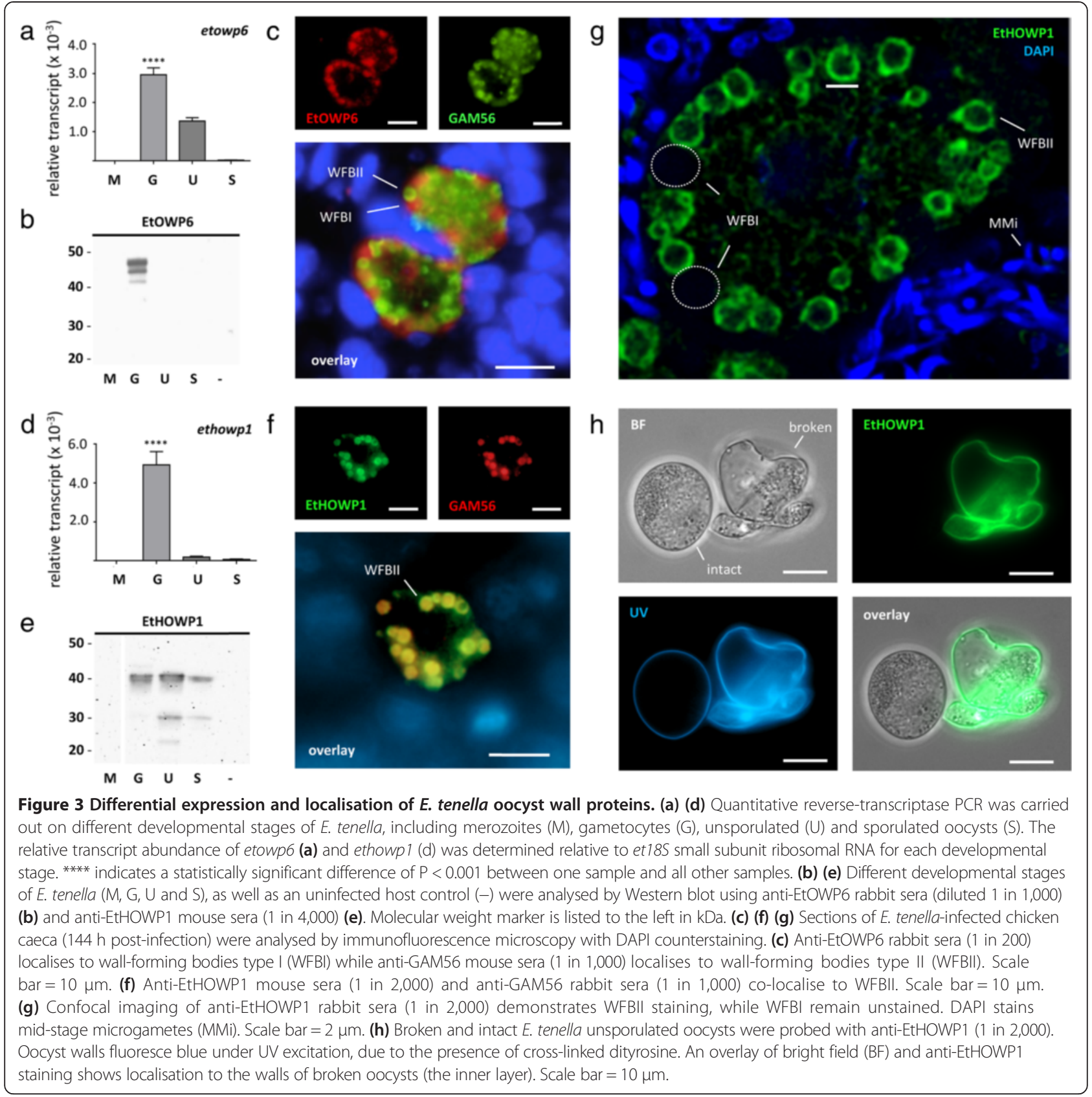

the processes accompanying their incorporation into this bilayered structure are also upregulated during gametocyte development (Table 2). GAM56, for example, undergoes proteolysis into smaller tyrosine-rich peptides, prior to oocyst wall assembly [30]. In the present study, upregulated gametocyte transcripts coding for a number of different proteases were identified including; one serine metalloprotease precursor, one aminopeptidase, an aspartic protease (eimepsin 2) and seven subtilisins. Subtilisins are responsible for processing tyrosine-rich proteins during $E$. tenella gametocyte development [18]. Additional analysis of three subtilisins, etsub1, etsub2 and etsub4, demonstrated that etsub2 is the most specific to gametocytes (Figure 4a); etsub1 transcript abundance was significantly higher in gametocytes but was still present in oocyst stages, while etsub4 was significantly higher in unsporulated oocysts than in gametocytes. Western blot analysis using anti-EtSUB2 polyclonal antibody detected a protein of approximately $60 \mathrm{kDa}$ in E. tenella gametocytes only (Figure $4 \mathrm{~b}$ ); the predicted size of $48.3 \mathrm{kDa}$ is probably an underestimate due to the truncated gene model of ETH_00025145. Furthermore, immunolocalisation studies using the same antibody revealed localisation of this serine protease to E. tenella macrogametocytes (Figure 4c), 
Table 2 Upregulated gametocyte transcripts coding for proteins involved in the biosynthesis of the oocyst wall

\begin{tabular}{|c|c|c|c|}
\hline Gene ID & FPKM & Annotation & Function \\
\hline ETH_00005950 & 651.50 & serine metalloprotease precursor & protease \\
\hline ETH_00013105 & 319.88 & aminopeptidase N 1 & protease \\
\hline ETH_00007420 & 113.15 & Eimepsin 2 (aspartic protease) & protease \\
\hline ETH_00000180 & 76.59 & subtilisin-like serine protease & protease \\
\hline ETH_00006825 & 69.68 & subtilisin 4 & protease \\
\hline ETH_00009270 & 59.38 & subtilisin-like serine protease & protease \\
\hline ETH_00000395 & 59.33 & subtilisin-like serine protease & protease \\
\hline ETH_00042675 & 41.92 & subtilisin sub8 & protease \\
\hline ETH_00025145 & 39.13 & subtilisin 2 & protease \\
\hline ETH_00009790 & 31.77 & subtilisin 1 & protease \\
\hline ETH_00034420 & 722.78 & thioredoxin & oxidoreductase \\
\hline ETH_00033360 & 475.71 & gmc oxidoreductase & oxidoreductase \\
\hline ETH_00032305 & 438.54 & peroxidoxin 2 & oxidoreductase \\
\hline ETH_00007785 & 343.29 & alkyldihydroxyacetonephosphate synthase, peroxisomal & oxidoreductase \\
\hline ETH_00025705 & 332.68 & thioredoxin & oxidoreductase \\
\hline ETH_00028385 & 311.75 & amiloride-sensitive amine oxidase, copper-containing & oxidoreductase \\
\hline ETH_00027865 & 282.95 & peroxiredoxin 3 & oxidoreductase \\
\hline ETH_00027760 & 79.18 & mannitol 2-dehydrogenase & oxidoreductase \\
\hline ETH_00015660 & 153.33 & UDP-glucose 4-epimerase & glycosylation \\
\hline ETH_00007235 & 58.24 & oligosaccharyl transferase STT3 & glycosylation \\
\hline ETH_00019125 & 52.30 & glucosamine-fructose-6-phosphate aminotransferase & glycosylation \\
\hline ETH_00005370 & 52.07 & UDP-N-acetyl-D-galactosamine:polypeptide N-acetylgalactosaminyltransferase T3 & glycosylation \\
\hline ETH_00007230 & 44.54 & dolichyl-diphosphooligosaccharide-protein glycosyltransferase subunit stt3a & glycosylation \\
\hline ETH_00018780 & 44.49 & beta-n-acetylglucosaminyltransferase-like protein & glycosylation \\
\hline ETH_00004905 & 653.67 & GNS1/SUR4 family & fatty acid metabolism \\
\hline ETH_00007660 & 497.09 & acyl-CoA-binding protein & fatty acid metabolism \\
\hline ETH_00032045 & 251.30 & acyl-coenzyme A oxidase & fatty acid metabolism \\
\hline ETH_00035050 & 179.62 & diacylglycerol acyltransferase & fatty acid metabolism \\
\hline ETH_00019415 & 135.48 & lipase class 3 & fatty acid metabolism \\
\hline ETH_00019675 & 113.45 & very long-chain acyl-CoA synthetase & fatty acid metabolism \\
\hline ETH_00029525 & 103.42 & acyl-CoA-binding protein & fatty acid metabolism \\
\hline ETH_00011255 & 101.30 & acyl-protein thioesterase 1 -like & fatty acid metabolism \\
\hline ETH_00025420 & 62.41 & peroxisomal multifunctional enzyme & fatty acid metabolism \\
\hline ETH_00015480 & 53.04 & EtPKS1 (polyketide synthase) & fatty acid metabolism \\
\hline ETH_00015230 & 23.12 & peroxisomal 2,4-dienoyl CoA reductase & fatty acid metabolism \\
\hline ETH_00005790 & 12.53 & EtPKS2 (polyketide synthase) & fatty acid metabolism \\
\hline ETH_00015485 & 11.34 & EtPKS1 (polyketide synthase) & fatty acid metabolism \\
\hline ETH_00005575 & 8.44 & enoyl-CoA hydratase/isomerase family protein & fatty acid metabolism \\
\hline ETH_00043845 & 8.02 & polyketide synthase & fatty acid metabolism \\
\hline ETH_00005785 & 3.72 & EtPKS2 (polyketide synthase) & fatty acid metabolism \\
\hline
\end{tabular}

Upregulated gametocyte transcripts coding for proteins with either a known or putative role in oocyst wall biosynthesis are listed along with their transcript abundance (FPKM), annotation and biological function. 


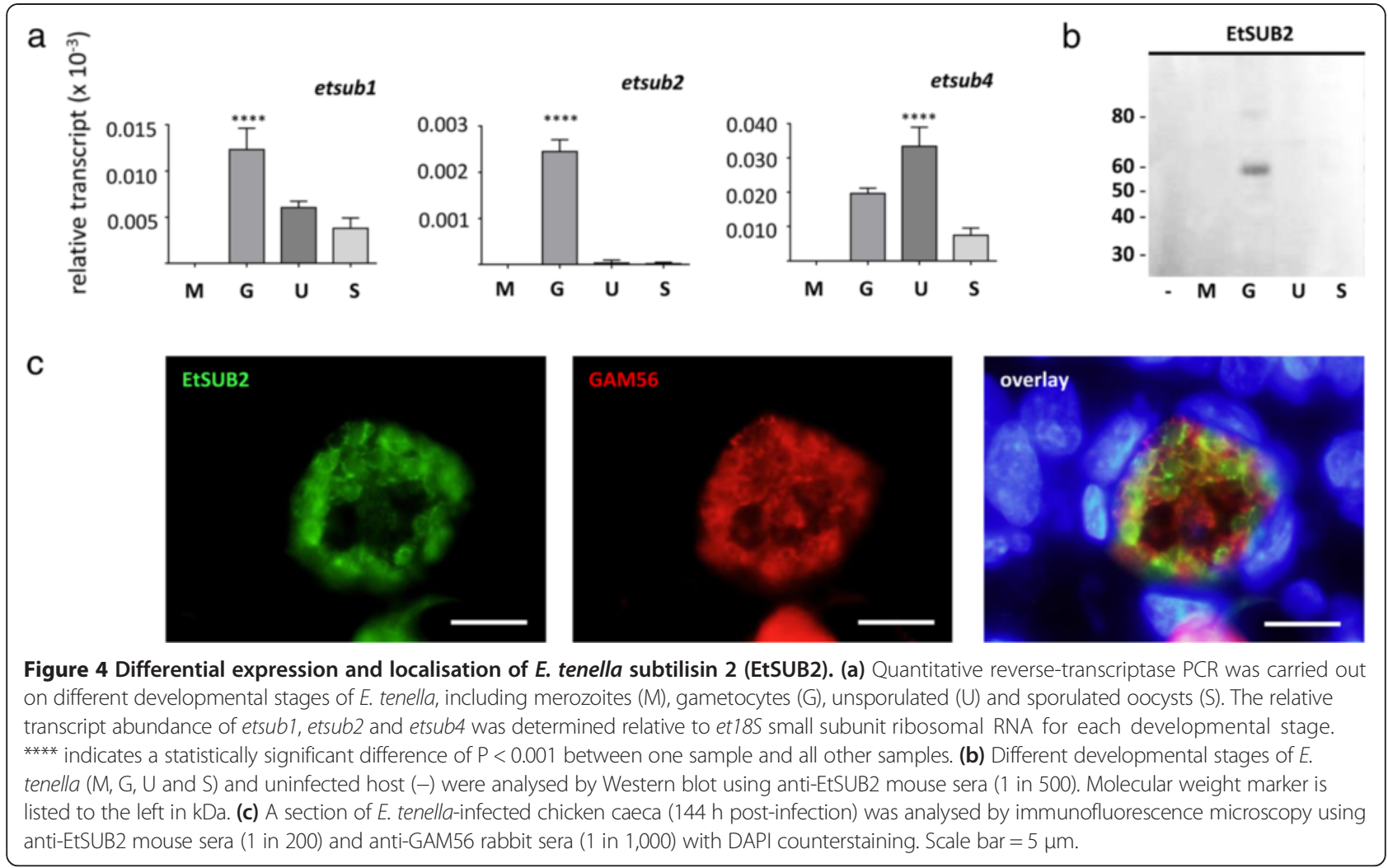

specifically to the periphery of the parasite cell and adjacent to WFBII (labelled with anti-GAM56).

Cross-linking of the smaller tyrosine-rich proteins into dityrosine imparts further stability to the oocyst wall and evidence implicates the role of peroxidases in catalysing this reaction [33]. Table 2 includes two peroxiredoxins (peroxidases), in addition to six genes coding for proteins with predicted oxidoreductase activities, potentially capable of cross-linking dityrosine. The amiloride-sensitive amine oxidase, copper-containing protein (EtAO2, coded by ETH_00028385) is of particular interest since its possession of an extracellular MAM (meprin, A5, $\mu$ )-domain [34] could place it at the site of oocyst wall biogenesis. Quantitative RT-PCR confirmed etao2 as an upregulated gametocyte transcript (Figure 5a). However, Western blot analysis using anti-EtAO2 polyclonal antibody detected a single $120 \mathrm{kDa}$ protein in mature gametocytes and unsporulated oocysts (Figure 5b). Immunolocalisation studies with this antibody reveal that EtAO2 localises to macrogametes (Figure 5c). Although EtAO2 appears to localise as foci within the cytoplasm, the staining pattern is distinct from the WFBI or WFBII. Aside from some augmented staining at the periphery of mature macrogametes (Figure 5c), there was no observable staining to oocyst stages in tissue sections as might be expected of for an enzyme involved in oocyst wall assembly. It should be noted, however, that given the expected size of EtAO2 is predicted at $183 \mathrm{kDa}(\sim 60 \mathrm{kDa}$ larger than observed by Western blot), some caution must be taken in interpreting results obtained with this antibody.

GAM56 and GAM82 have both been shown to be heavily glycosylated [35], a process that is linked to a co-regulated glycosylation pathway in Eimeria [17]. In the present study, six upregulated gametocyte transcripts were identified that code for enzymes in the protein glycosylation pathway, including EtGFAT (glucosamine: fructose6-phophate aminotransferase) (ETH_00019125), the primary enzyme in the amino sugar biosynthesis pathway that was previously localised to E. tenella macrogametes [17]. Interestingly, recent studies have indicated the coccidian oocyst wall architecture is comprised not only of glycoproteins but also interlayered $\beta$-1,3-glucan fibrils [36] and an outer layer of acid-fast lipids [37]. Glucan synthase, the enzyme catalysing the synthesis of $\beta$-1,3-glucan fibrils, is coded by the gene ETH_00000330 whose transcript levels were upregulated 14 -fold in E. tenella gametocytes compared to merozoites (note, ETH_00000330 was not strictly listed as an 'upregulated gametocyte transcript' since gametocyte transcript abundance was only four-fold higher than that of sporozoites). A total of sixteen upregulated gametocyte transcripts code for proteins with predicted roles in synthesising or remodelling the acid-fast lipid layer of the E. tenella oocyst wall [37], including the previously described polyketide 
a

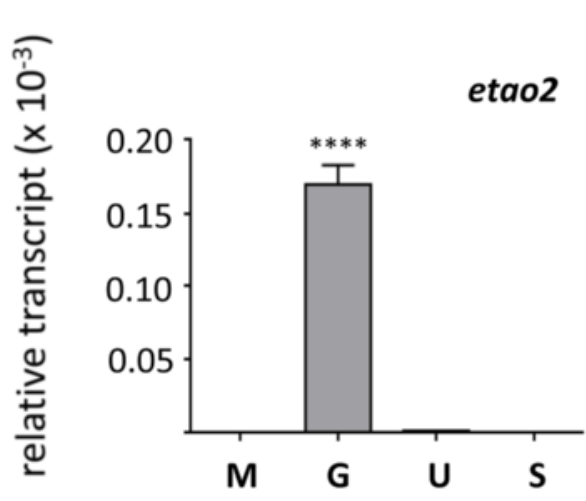

b

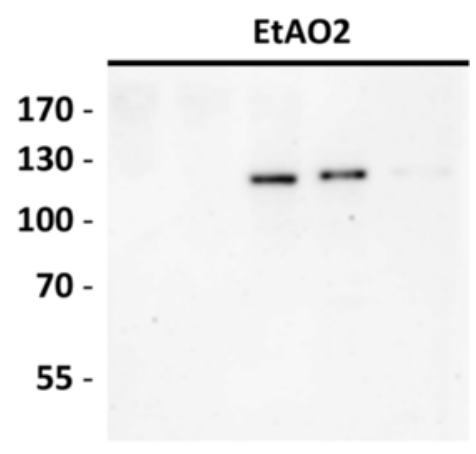

- M G U S
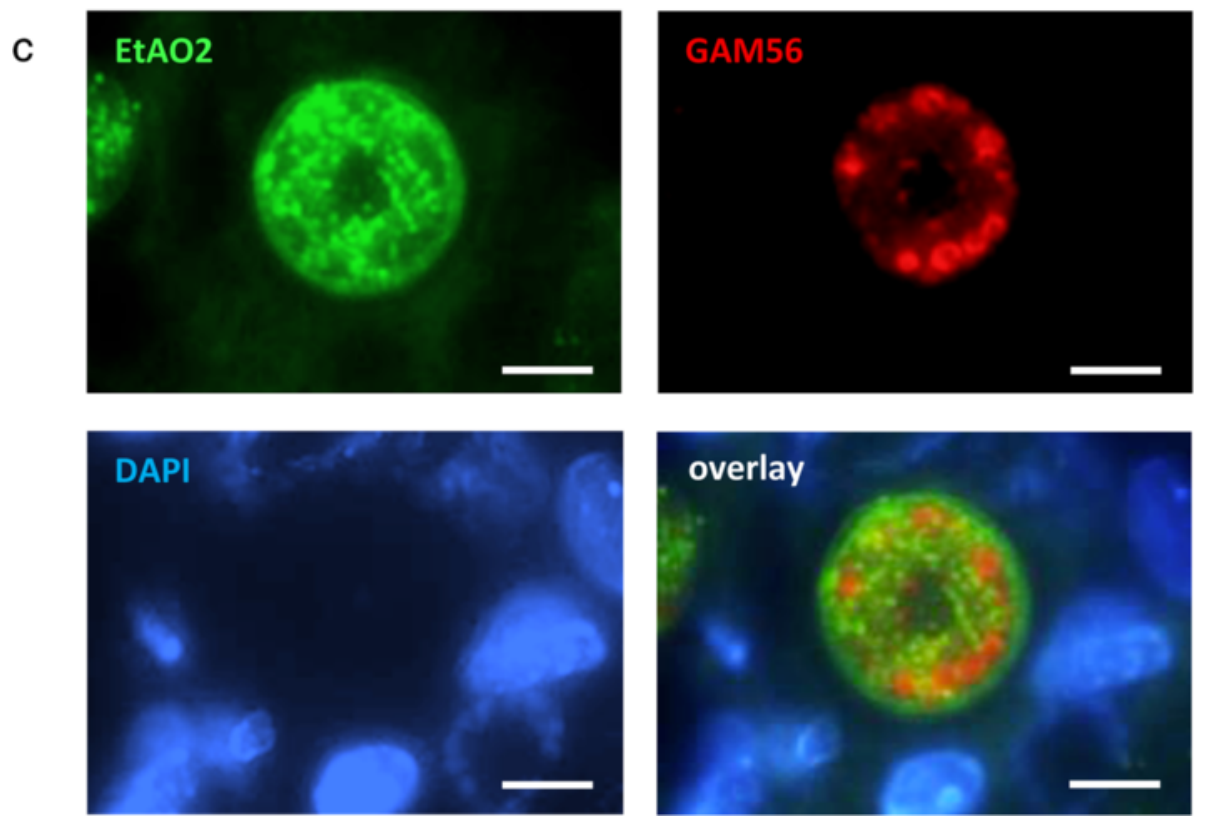

Figure 5 Differential expression and localisation of $E$. tenella amine oxidase 2 (EtAO2). (a) Quantitative reverse-transcriptase PCR was carried out on different developmental stages of $E$. tenella, including merozoites (M), gametocytes (G), unsporulated (U) and sporulated oocysts (S). The relative transcript abundance of eta02 was determined relative to et18S small subunit ribosomal RNA for each developmental stage. **** indicates a statistically significant difference of $\mathrm{P}<0.001$ between one sample and all other samples. (b) Different developmental stages of E. tenella ( $M, G, U$ and $S)$ and uninfected host (-) were analysed by Western blot using anti-EtAO2 mouse sera (1 in 1,000). Molecular weight marker is listed to the left in $\mathrm{kDa}$. (c) A section of $E$. tenella-infected chicken caeca (144 h post-infection) was analysed by immunofluorescence microscopy using anti-EtAO2 mouse sera (1 in 500) and anti-GAM56 rabbit sera $(1$ in 1,000) with DAPI counterstaining. Scale bar $=5 \mu \mathrm{m}$.

synthases, EtPKS1 (predicted from both ETH_00015480 and ETH_00015485) and EtPKS2 (ETH_00005790 and ETH_00005785). Note, the upregulated gametocyte transcript ETH_00043845 appears to code for an additional, as yet undescribed PKS.

Targeted inhibition of highly-divergent, parasite-specific biochemical pathways is yet another strategy for combating coccidian transmission; inhibition of glucan synthase has shown promise in reducing oocyst output in E. tenella [36]. The potential to block oocyst wall formation by inhibition of gametocyte-specific subtilisins and oxidoreductases warrants further investigation.
Microgamete biology is underscored by numerous gametocyte targets, including the gamete fusion protein EtHAP2, which localises to $E$. tenella microgametes

Structural studies of E. tenella microgametes describe terminally differentiated stages comprised of a nucleus, a mitochondrion, a number of longitudinally running microtubules and two flagella (reviewed in [4]); in many respects their biology is analogous to that of eukaryotic sperm. While characterisation of microgamete-specific markers in the Coccidia is limited, proteomic analyses of purified microgametes in the related apicomplexan, Plasmodium berghei, has highlighted an important role 
for proteins involved in DNA replication and the axoneme, a specialised cytoskeletal structure of eukaryotic flagella [38,39]. In the present study, genes coding for highly-conserved axoneme-associated proteins, including twenty dyneins, two radial spoke head proteins, two basal body proteins and one central apparatus protein were identified as upregulated gametocyte transcripts (Table 3). ETH_00013995, the most abundant of these microgamete-specific transcripts, codes for an armadillo/ beta-catenin-like repeat protein that is a homologue of PF16, a microgamete protein of Plasmodium falciparum. PF16 is a component of the axoneme central apparatus and is essential for microgamete motility and fertilisation [40]. Likewise, ETH_00022085 shows homology to a protein, TAX-2 (Trypanosome Axonemal Protein-2), required for motility in the flagellated parasite, Trypanosoma brucei [41]. Also listed in Table 3 are genes coding for an additional eight flagellar-associated proteins and for proteins with predicted non-flagellar functions. Protamine (ETH_00009265), for instance, is a histone-like protein that binds sperm DNA, condensing the genome into an inactive state [42], while calmegin (ETH_00022665) is a testis-specific ER protein that functions as a protein chaperone and is required for fertility [43,44]. AAT-1 (AMY-1-associated protein expressed in testes-1) protein (ETH_00005660) is predicted to have versatile functions in spermatogenesis, including the modulation of energy required for fertilisation [45], while enkurin (ETH_00003635) is expressed in mammalian sperm and is thought to be involved in signal transduction pathways of fertilisation during gamete fusion [46].

Finally, two upregulated gametocyte transcripts, ETH_00017050 and ETH_00017055, are tandemly located in the E. tenella genome and code for proteins with homology to a family of transmembrane proteins, known as HAP2-GCS1 (HAPLESS2/Generative Cell Specific) whose expression is restricted to male gametes in a range of different organisms [47]. Each of these genes aligns to different regions of an E. tenella 'GCS1' mRNA sequence (GenBank: AB723702.1, direct submission, unpublished) implying a misprediction of both ETH_00017050 and ETH_00017055 exon models (data not shown). Mapping of gametocyte RNA Seq reads to the E. tenella genome supported that the two HAP2-like genes are actually part of one contiguous gene transcript (Figure 6a); in fact, the canonical HAP2 domain is located in what is currently predicted as an intergenic region of the E. tenella genome, highlighting the current pitfalls in gene prediction models not informed by transcriptome data. Subsequent RT-PCR and sequencing of the ethap 2 transcript confirmed this observation and revealed 100\% of identity to the GenBank mRNA sequence, herein used as the EtHAP2 CDS.

The expression of HAP2 in Plasmodium is restricted to microgametes and is required for gamete fusion and subsequent fertilisation [48-50]. The HAP2 domains of EtHAP2 and PbHAP2 are partially conserved (33\% sequence identity), with further sequence identity being observed in the HAP2 domains of CrHAP2 (from the green alga Chlamydomonas reinhardtii; 27\%) [48] and a putative TgHAP2 (T. gondii; 42\%) (Figure 6b). Although sequence homology outside this domain is limited, the general architecture of the EtHAP2 protein, including the presence of a signal peptide, extracellular HAP2 domain, single transmembrane domain and an intracellular, positively-charged (lysine/arginine-rich) C-terminal region (Figure 6c), is as described previously for HAP2-GSC1 proteins [51].

The extracellular HAP2 domain is thought to act as a fusogen, mediating membrane fusion between mating gametes [47]. Antibodies raised against the HAP2 domain of EtHAP2 reacted specifically with microgametes of E. tenella, with no observable reactivity with either macrogametes or developing oocysts (Figure 7a). EtHAP2 staining appeared more intense in late-stage (mature) microgametes than either mid-stage or early-stage microgametes, consistent with a predicted role in fertilisation. Efforts to determine the subcellular localisation of EtHAP2 revealed a distribution pattern distinct from that of the microgamete nucleus and mitochondrion, organelles that appear as near-continuous, DAPI-positive structures (Figure 7b). Beyond this, it was difficult to confirm whether EtHAP2 localisation was cytoplasmic, surface or, less likely, as part of the flagella. In the flowering plant, Arabidopsis thaliana, HAP2 redistributes from the cytoplasm to the surface of sperm cells upon contact with a cysteine-rich protein and potential ligand, EC-1 (Egg Cell 1) [52]. Likewise, it is conceivable that EtHAP2 is exposed at the surface of microgametes only after attachment to macrogametes during fertilisation.

Evidence suggests that unfertilised Eimeria macrogametes fail to develop into mature oocysts and/or are non-infective due to a failure to sporulate $[53,54]$. Therefore, disturbing the biological processes of microgametes, including motility and gamete fusion, is another promising strategy to block the transmission of apicomplexan parasites.

\section{HOWP1 and AO2 are macrogametocyte proteins that are conserved in $T$. gondii}

The fundamental biological processes of sexual stage development and oocyst formation are broadly conserved in the Coccidia. It would be expected, therefore, that many of the E. tenella gametocyte proteins identified in the present study would be biologically conserved in T. gondii. Indeed, sequence homology searching of the $T$. gondii genome database (www.toxodb.org) revealed that TGME49_316890 (referred to herein as TgHOWP1) may be the structural orthologue of EtHOWP1, although the two proteins share only $24.7 \%$ sequence identity (and a 
Table 3 Upregulated gametocyte transcripts coding for microgamete proteins

\begin{tabular}{|c|c|c|c|}
\hline Gene ID & FPKM & Annotation & Function \\
\hline ETH_00013995 & 223.15 & armadillo/beta-catenin-like repeat protein & axoneme \\
\hline ETH_00014050 & 195.46 & radial spoke head & axoneme \\
\hline ETH_00014055 & 184.22 & radial spoke head protein 9 homolog & axoneme \\
\hline ETH_00025255 & 125.32 & dynein intermediate chain, putative & axoneme \\
\hline ETH_00008420 & 99.32 & flagellar outer dynein arm light chain 2, putative & axoneme \\
\hline ETH_00002085 & 81.54 & ciliary basal body-associated, B9 protein & axoneme \\
\hline ETH_00023565 & 60.23 & flagellar inner arm dynein 1 heavy chain beta & axoneme \\
\hline ETH_00008820 & 50.32 & axonemal dynein gamma heavy & axoneme \\
\hline ETH_00008815 & 45.22 & axonemal dynein gamma heavy & axoneme \\
\hline ETH_00017815 & 30.16 & dynein heavy & axoneme \\
\hline ETH_00008810 & 29.97 & axonemal dynein gamma heavy & axoneme \\
\hline ETH_00022085 & 28.85 & cytochrome b5 domain-containing protein 1-like & axoneme \\
\hline ETH_00027240 & 23.02 & flagellar inner arm dynein 1 heavy chain alpha & axoneme \\
\hline ETH_00023560 & 22.57 & dynein heavy chain axonemal & axoneme \\
\hline ETH_00022700 & 20.51 & dynein heavy chain axonemal & axoneme \\
\hline ETH_00008805 & 20.26 & dynein heavy chain family protein & axoneme \\
\hline ETH_00023555 & 18.16 & dynein heavy & axoneme \\
\hline ETH_00043205 & 16.08 & dynein heavy chain axonemal-like & axoneme \\
\hline ETH_00008800 & 14.75 & dynein heavy chain & axoneme \\
\hline ETH_00023545 & 14.07 & flagellar inner arm dynein 1 heavy chain beta & axoneme \\
\hline ETH_00008795 & 13.24 & flagellar outer dynein arm heavy chain gamma & axoneme \\
\hline ETH_00027230 & 12.44 & dynein heavy chain & axoneme \\
\hline ETH_00017810 & 11.97 & dynein heavy chain family protein & axoneme \\
\hline ETH_00039340 & 11.06 & flagellar basal body protein & axoneme \\
\hline ETH_00027220 & 9.11 & flagellar inner arm dynein 1 heavy chain alpha & axoneme \\
\hline ETH_00027225 & 7.60 & flagellar inner arm dynein 1 heavy chain alpha & axoneme \\
\hline ETH_00030810 & 115.85 & flagellar associated protein & flagella \\
\hline ETH_00023960 & 55.80 & flagellar associated protein & flagella \\
\hline ETH_00013980 & 53.78 & flagellar associated protein & flagella \\
\hline ETH_00023965 & 50.49 & flagellar associated protein & flagella \\
\hline ETH_00019365 & 42.87 & flagellar associated related & flagella \\
\hline ETH_00004845 & 41.61 & flagellar associated protein & flagella \\
\hline ETH_00019370 & 31.03 & flagellar associated related & flagella \\
\hline ETH_00002895 & 22.43 & testis-expressed sequence 9 protein & flagella \\
\hline ETH_00009265 & 53.51 & protamine $\mathrm{P} 1$ protein, putative & DNA condensation \\
\hline ETH_00003635 & 232.25 & enkurin-related protein & gamete fusion \\
\hline ETH_00017050 & 107.31 & generative cell specific-1 (HAP2) & gamete fusion \\
\hline ETH_00017055 & 102.53 & generative cell specific-1 (HAP2) & gamete fusion \\
\hline ETH_00022665 & 71.08 & calmegin, putative & protein chaperone \\
\hline ETH_00005660 & 64.65 & AMY-1-associated protein expressed in testes & energy \\
\hline
\end{tabular}

Upregulated gametocyte transcripts coding for proteins with a putative role in microgamete biology are listed along with their transcript abundance (FPKM), annotation and biological function.

predicted signal peptide) (Additional file 8). Importantly, TgHOWP1 was identified previously in a proteomic analysis of purified T. gondii oocyst walls [55]. In the present study, polyclonal antisera raised against a TgHOWP1-GST fusion protein specifically stained $T$. gondii macrogametes in sections of infected cat intestine, while merozoites 


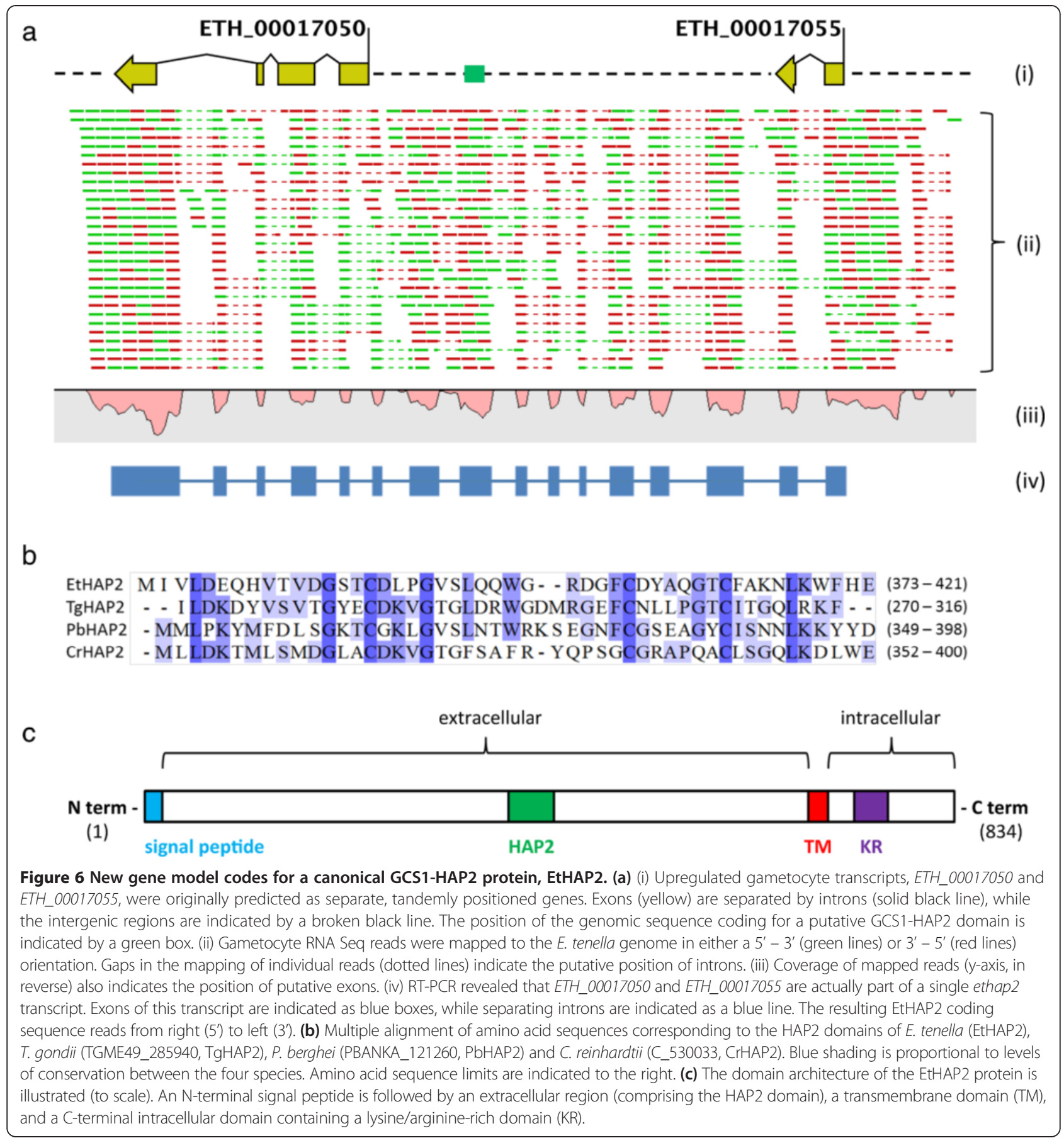

(contained within late schizonts) remained unstained (Figure 8a). TgHOWP1 appeared to localise to organelles resembling wall forming bodies, although the lack of a WFB marker in $T$. gondii makes this observation somewhat presumptive.

A putative orthologue of EtAO2 was also identified in $T$. gondii (TgAO2), although like EtHAP2, the protein is predicted to be encoded by two adjacent genes, TGME49_286782 and TGME49_286778. Preliminary data from RNA Seq analysis of $T$. gondii gametocytes (unpublished observations) confirms that these two exon models actually represent a single tgao2 gene transcript, as predicted in a previous version of the T. gondii genome assembly (TGME49_ 086780). The protein sequences of EtAO2 and TgAO2 (using the TGME49_086780 model) share 59.7\% sequence identity, although this extends to $69.8 \%$ for the extracellular MAM domain and $70.5 \%$ for the copper amine oxidase domain 
a
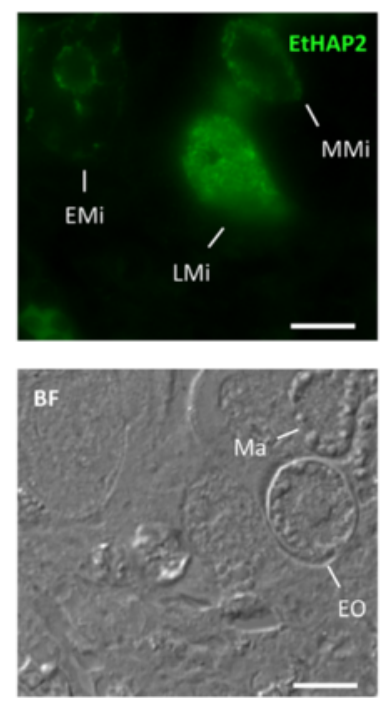
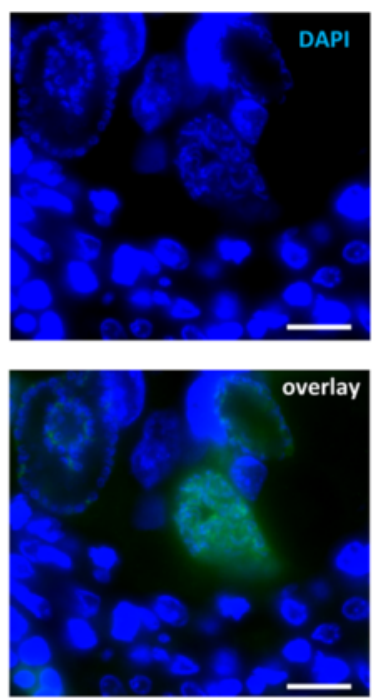

b

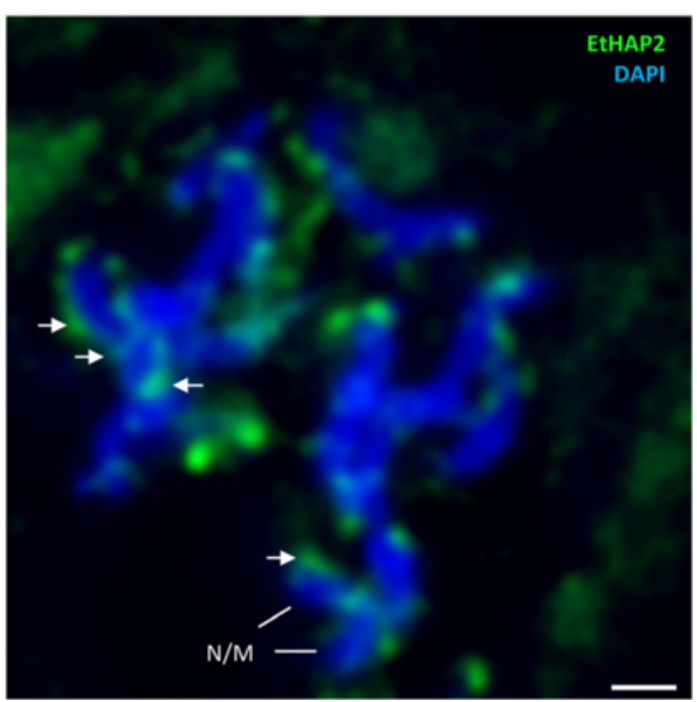

Figure 7 EtHAP2 expression is restricted to $E$. tenella microgametes. (a) Sections of E. tenella-infected chicken caeca (144 h post-infection) were stained with anti-EtHAP2 mouse sera (1 in 100) and DAPI. EtHAP2 (green) localised with increasing intensity to DAPI-positive early-, mid- and late-stage microgametocytes (EMi, MMi and LMi, respectively). Macrogametes (Ma) and early oocysts (EO), visible under bright field (BF), were EtHAP2-negative. Scale bar $=10 \mu \mathrm{m}$. (b) The same tissue section was imaged using confocal microscopy and post-acquisition deconvolution. The nucleus and mitochondrion (N/M) of individual late-stage microgametes stain DAPI-positive and appear continuous and elongated. EtHAP2 (green) localisation (arrows) is distinct but often adjacent to N/M, consistent with either cytosolic or surface localisation. Scale bar = 1 um.

(Additional file 9). Antibody raised against a TgAO2-GST fusion protein corresponding to the entire MAM domain reacted specifically against $T$. gondii macrogametes, like EtAO2, localising to distinct cytoplasmic foci (Figure $8 \mathrm{~b}$ ). In contrast to EtAO2, additional staining was also observed on the surface of early oocysts (Figure $8 \mathrm{c}$ ), consistent with a possible role for $\mathrm{TgAO} 2$ in cross-linking tyrosine-rich proteins in the developing oocyst wall of $T$. gondii.

\section{Conclusions}

In this study, a comparative RNA Seq transcriptomics approach led to the identification of hundreds of genes specifically expressed in E. tenella gametocytes. This data set represents a snapshot of the mechanisms at play in coccidian sexual biology and also highlights previously undescribed transmission blocking targets - in particular, two oocyst wall proteins, a subtilisin, an oxidoreductase and a microgamete-specific gamete fusion protein. With so many hypothetical genes identified, further functional characterisation will be paramount for determining the key players in distinct sexual stage processes, including microgamete motility, fertilization and oocyst wall biogenesis. As the transmission blocking potential of these Eimeria targets are assessed in future studies, wider implications can be expected in related Coccidia, like Toxoplasma, where transmission blocking remains a relatively unexplored control strategy.

\section{Methods}

\section{Parasites}

One day-old chicks (Australorp; Barter and Sons Hatchery, Luddenham, Australia) were housed at the Ernst Facility Animal House (University of Technology, Sydney), under heat lamps for 2 weeks and at $21^{\circ} \mathrm{C}$ thereafter, with a 12-hour light/dark cycle with free access to food and water. To confirm chickens as free of infection prior to experimental inoculation, faeces were analysed by salt flotation and light microscopy to ensure the absence of oocysts [56]. Chickens were infected orally at 4 to 5 week old chickens were used of age with $2.5 \times 10^{3}$ sporulated oocysts of Eimeria tenella (Houghton strain, originally provided by Janene Bumstead, Institute for Animal Health, Compton, UK). Fresh E. tenella oocysts were harvested 7 days post-infection (p.i.) from the caeca following protocols described previously [57]. Oocyst sporulation was carried out at $28^{\circ} \mathrm{C}$ for $72-120 \mathrm{~h}$ using a lowpressure aquarium pump for aeration. Sporulated oocysts were then treated with $2.8 \mathrm{M} \mathrm{NaCl}$ and $2 \%$ sodium hypochlorite (Milton solution) and stored in $2 \%$ potassium dichromate at $4{ }^{\circ} \mathrm{C}$. Unsporulated oocysts were also treated with Milton solution and stored at $-80^{\circ} \mathrm{C}$. Merozoites (112 h p.i.) and gametocytes (134 $\mathrm{h}$ and $144 \mathrm{~h}$ p.i.) were isolated from infected chicken caeca following techniques published previously $[23,56]$. Sporozoites were excysted from purified sporulated oocysts as described previously [58]. Whole caecal samples were taken at $144 \mathrm{~h}$ p.i. for immunolocalisation studies. 
a
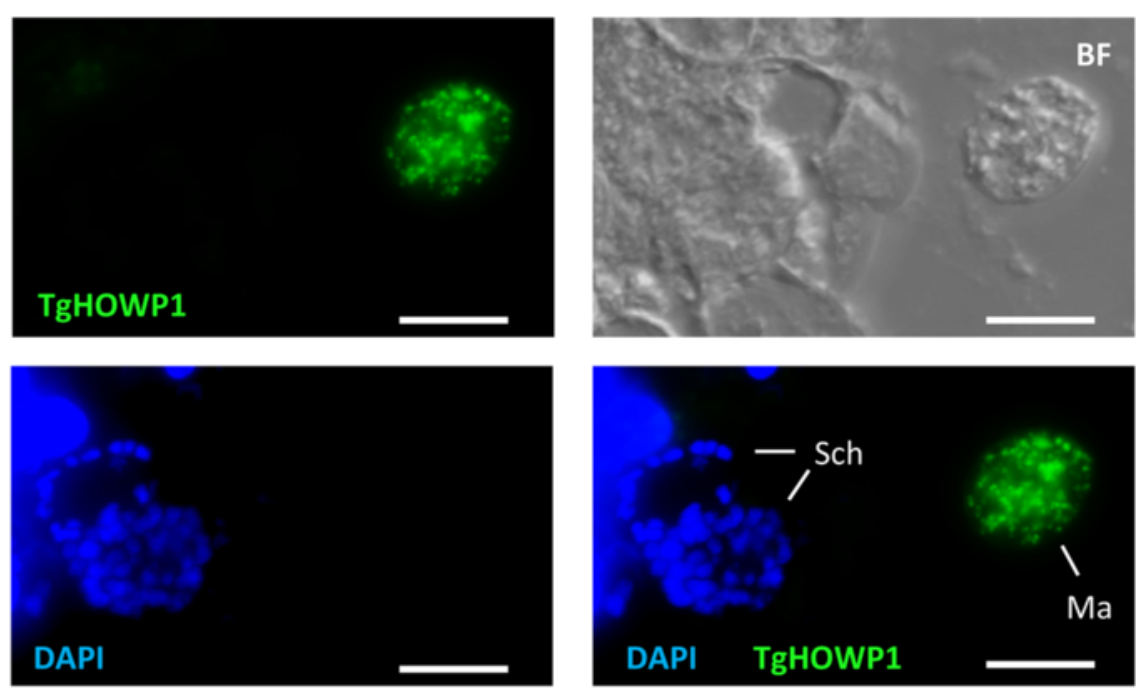

b
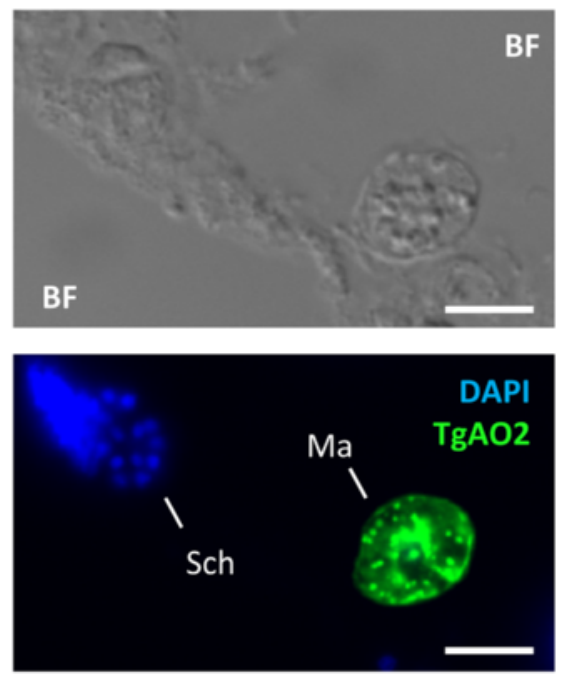

C
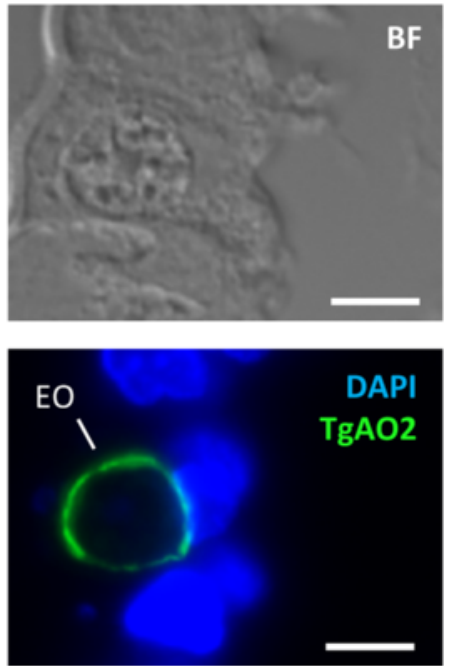

Figure 8 TgHOWP1 and TgAO2 localisation in T. gondii-infected cat intestine. Sections of T. gondii-infected cat intestine (day 7 post-infection) were analysed by immunofluorescence microscopy with DAPI counterstaining. (a) Anti-TgHOWP1 mouse sera (1 in 100) localised to T. gondii macrogametes (Ma) but not to DAPI-positive late schizonts (Sch). Scale bar $=5 \mu \mathrm{m}$. (b) Anti-TgAO2 (1 in 100) localised to T. gondii macrogametes (Ma) but not to DAPI-positive late schizonts (Sch). Scale bar $=5 \mu \mathrm{m}$. (c) Anti-TgAO2 also localised to early oocysts (EO). Scale bar $=5 \mu \mathrm{m}$.

The samples were stored in $2 \%$ paraformaldehyde/0.1 M phosphate buffer (pH 7.4) and embedded in paraffin.

Paraffin blocks of cat intestine infected with the type II CZ T. gondii isolate, originally isolated from the faeces of a captive Siberian tiger (Panthera tigris altaica) at the Dvůr Králové Zoo (Czech Republic); parasites were passed once through mice to produce bradyzoite-containing tissue cysts and once through cats to generate $\sim 50$ million CZ oocysts, which were used to infect four sheep. Brain tissue from sheep containing bradyzoites in tissue cysts was fed to cats. At day 7 post-infection, the small intestine was removed, stored in $4 \%$ formaldehyde/
0.1 M phosphate buffer ( $\mathrm{pH} 7.4$ ) and embedded in paraffin for future immunolocalisation experiments.

\section{Total RNA preparation}

Parasite samples were resuspended in $\mathrm{Trizol}^{\circ}$ Reagent (Invitrogen) for total RNA extraction. Merozoite, gametocyte and excysted sporozoite samples were homogenised by repeated pipetting while unsporulated and sporulated oocysts were homogenised by vortexing with an equal volume glass beads $(710-1,180 \mu \mathrm{m}$, Sigma) until oocyst and sporocyst walls were completely broken (no more than ten cycles of $1 \mathrm{~min}$ on, 1 min off). Total 
RNA was purified from homogenised samples as per manufacturer's instructions. Quantification of total RNA was carried out using a Qubit fluorometer (Invitrogen) Assessment of total RNA quality was carried out on a Bioanalyzer 2100 (Agilent) using an Agilent RNA 6000 Pico Kit. Prior to either RNA Seq or cDNA synthesis, total RNA was treated with RNase-free DNase I (Qiagen) on an RNeasy column (Qiagen), re-purified and, in the case of RNA Seq, re-assessed on a Bioanalyzer 2100.

\section{cDNA library construction and RNA Seq}

Genome-wide transcriptome libraries were produced from triplicate biological replicates of $E$. tenella merozoites, gametocytes and sporozoites. Approximately $200 \mathrm{ng}$ of mRNA were enriched from 15-20 $\mu \mathrm{g}$ of total RNA using the TruSeq RNA Sample Preparation Kit (Illumina). cDNA synthesis and amplification for unstranded, paired-end sequencing was carried out using the Encore Complete RNA-Seq Library Systems kit (NuGEN). cDNA libraries were selected for fragment sizes of between 150-250 bp and amplified for 15-18 cycles according to the Encore ${ }^{\circ}$ protocol. Library size and concentration were assessed using a Bioanalyzer 2100 (Agilent) and a Qubit fluorometer (Invitrogen), respectively. Transcriptome libraries were sequenced using the HiSeq 2000 Sequencing System (Illumina). Each library was run on two lanes in a flow cell in order to maximise the total number of RNA Seq reads. The combined RNA Seq run resulted in nine separate fastq files representing each library, containing sequenced paired reads and quality control data.

For mapping, individual reads were aligned to the $E$. tenella Houghton strain genome version 8.1 (available on www.toxodb.org) of the recently published E. tenella (Houghton strain) genome sequence [59] using TopHat2 [60]. The counts of reads mapped to gene models were processed by Bioconductor library DESeq [19], producing a table that includes the total mapped reads per gene model for each library, in addition to the normalised read count per gene model for each biological stage (averaged from biological replicates). Fold changes, $\log 2$ fold-changes, p-values and adjusted p-values (adjusted for multiple testing with the Benjamini-Hochberg procedure which controls for false discovery rate [61]) are also reported. In the present study, a fold change of equal to or greater than eight ( $\log 2$ fold change $\geq 3$ ) was used to identify differentially expressed genes, excluding false positives represented by a p-adjusted value cut-off of 0.05 or over. Pair-wise hierarchical clustering between the vectors of read counts of all pairs of samples was carried out using the heatmap. 2 function (available in the gplots package from CRAN, cran.r-project.org) according to default settings. An additional measurement of transcript abundance, FPKM (fragments per kilobase of exon model per million mapped reads), was calculated for each gene using the formula "FPKM $=10^{6} \times \mathrm{C} /(\mathrm{N} \times \mathrm{L})$ " where $\mathrm{C}$ is the number of paired-reads mapped to the exon model of a gene, $\mathrm{N}$ is the total number of mapped reads in each library and $\mathrm{L}$ is the length of the gene in kilobases.

\section{Quantitative reverse-transcriptase PCR (qRT-PCR)}

The cDNA samples were synthesised from DNase-treated total RNA using the Superscript ${ }^{\mathrm{TM}}$ III First-Strand Synthesis System (Invitrogen), using random hexamer primers. Quantitative RT-PCR was carried out on a RotorGene Q real-time PCR system (Qiagen). Individual reactions were prepared with $0.5 \mu \mathrm{M}$ of each primer, $5 \mathrm{ng}$ of cDNA and SYBR Green PCR master mix (Qiagen) to a final volume of $20 \mu \mathrm{l}$. All experiments were performed twice with separate biological replicates. For each experiment, reactions were performed in triplicate and expression of individual genes was normalised to $\mathrm{Ct}$ values of E. tenella small ribosomal subunit $18 S$ RNA using the delta Ct formula: $2^{-(\text {Ctgene }-\mathrm{Ctet18S)}}$. All primers were designed using Primer3 v. 0.4.0 (http://bioinfo.ut.ee/primer3-0.4.0/) and are listed in Additional file 10. Amplified products were sub-cloned into $\mathrm{pGEM}^{\circ}-\mathrm{T}$ Easy vector (Promega) as per manufacturer's instructions and sequenced with vectorspecific primers to confirm amplification of correct targets.

\section{DNA manipulation}

Coding sequences of different parasite genes were amplified by PCR from either genomic DNA or CDNA (indicated in Additional file 10) using Pfu DNA Polymerase (Thermo Scientific) according to manufacturer's instructions. The gene-specific primers used for amplification and subsequent cloning into pET41a $(+)$ are also listed in Additional file 10. All plasmids were sequenced with vector-specific primers to confirm correct insertion into pET41a(+).

\section{Recombinant protein expression}

The pET41a $(+)$ plasmid $\left(\right.$ Novagen $\left.^{\circ}\right)$ was used for the expression of truncated forms of EtHOWP1 (residues 20-301 of ETH_00018895), EtHAP2 (75 residues coded by the genomic region 210518-210294 of HG674888), TgHOWP1 (residues 24-407 of TGME49_316890) and TgAO2 (residues 176-532 of TGME49_086780) proteins, producing recombinant proteins with $\mathrm{N}$-terminal GST tags and flanking 6-His regions. An auto-induction protocol was followed for high-expression of GST-fusion proteins [62]. Briefly, plasmids were transformed into BL21 Escherichia coli and grown overnight in non-inducing, PG media at $37^{\circ} \mathrm{C}, 220 \mathrm{rpm}$. ZYP-5052 media was inoculated at 1 in 2,000 with overnight culture and grown for $20 \mathrm{~h}$ at $37^{\circ} \mathrm{C}, 220 \mathrm{rpm}$. A truncated form of EtSUB1 (residues 28-113 of ETH_00009790) and of a previous version of EtSUB2 (residues 456-685 of XM_001238654.1) were expressed with an C-terminal histidine tag in pET101/ ${\mathrm{D}-\mathrm{TOPO}^{\circ} \text { (Invitrogen }}^{\mathrm{m}}$, Australia) and a truncated form 
of EtAO2 (residues 44-1179 of ETH_00028385) was expressed with an N-terminal histidine tag in the E3 expression plasmid (GenScript USA Inc.) by standard IPTG induction in BL21 Escherichia coli. EtHOWP1-GST and TgHOWP1-GST were purified via their GST tags under native conditions using Glutathione Sepharose 4 Fast Flow (GE Healthcare) as per manufacturer's instructions and eluted in $30 \mathrm{mM}$ reduced GSH. EtHAP2-GST and TgAO2-GST were purified via their 6-His tags under denaturing conditions using NiNTA agarose (Qiagen) as per manufacturer's instructions and eluted in $250 \mathrm{mM}$ imidazole. EtSUB1, EtSUB2 and EtAO2 were purified via the 6-His tag under native conditions using the Profina ${ }^{\mathrm{TM}}$ protein purification system and Native IMAC Purification Kit (Bio-Scale Mini Profinity IMAC nickel-chelating column and desalting column) as per manufacturer's instructions. Purified proteins were dialysed overnight at $4^{\circ} \mathrm{C}$ in $25 \mathrm{mM}$ ammonium acetate using a Slide-A-Lyzer Dialysis Cassette, $10 \mathrm{~K}$ molecular weight cut-off (Thermo Scientific) and stored at $-20^{\circ} \mathrm{C}$.

\section{Antibodies}

Polyclonal sera was collected from mice immunised with recombinant forms of EtHOWP1-GST, EtHAP2-GST, TgHOWP1-GST, TgAO2-GST, EtSUB2 and EtAO2 using procedures described previously [30]. Polyclonal sera was also collected from rabbits immunised with a synthetic peptide (Auspep Pty. Ltd.) representing EtOWP6 (residues 164-179 of ETH_00012470) conjugated to KLH (keyhole limpet haemocyanin) carrier protein, using methods described previously [15]. Importantly, although the primary amino acid sequence of the recombinant EtSUB2 antigen is partially conserved with other E. tenella subtilisins, anti-EtSUB2 sera reactivity was shown by ELISA to be specific to the EtSUB2 antigen, not reacting significantly with any other subtilisin, including EtSUB1, with which it shares $37 \%$ identity. Briefly, for the assessment of antibody specificity by ELISA, recombinant protease antigens were diluted in ELISA buffer 1 to a final concentration of $5 \mathrm{ng} / \mathrm{ml}$, and $100 \mathrm{ml}$ aliquots (500 ng per well) added to a 96-well plate. Plates were incubated overnight at $4^{\circ} \mathrm{C}$ to allow the antigen to bind. The following day, plates were washed twice with PBS/0.05\% TWEEN20 ${ }^{\circ}$ and once with PBS to remove unbound antigen. Plates were then blocked with $200 \mathrm{ml}$ per well of PBS/5\% SMP for $1 \mathrm{~h}$ at room temperature with gentle agitation. Plates were then washed as before, and $100 \mathrm{ml}$ aliquots of primary antibody diluted in PBS/2.5\% SMP added to each well and incubated for $1 \mathrm{~h}$ at room temperature with gentleagitation. The plates were washed again as above and $100 \mu \mathrm{L}$ aliquots of secondary antibody (goat-derived, anti-rabbit or anti-mouse IgG, H\&L chain-alkaline phosphatase conjugate) diluted at 1:2000 in PBS/2.5\% SMP were added to eachwell. Plates were incubated for a further hour at room temperature, washed again and $200 \mu \mathrm{L}$ of $1 \mathrm{mg} / \mathrm{ml}$ p-nitrophenyl phosphate added to each well. The reaction was allowed to develop for $20 \mathrm{mins}$ at $37^{\circ} \mathrm{C}$, with a maximum of $60 \mathrm{~min}$, and absorbance was read at $405 \mathrm{~nm}$ using the FLUOstar Omega multi-detection microplate reader (BMG Labtech). All samples were tested in quadruplicate.

\section{Immunofluorescent microscopy}

Sections of $3 \mu \mathrm{m}$ were prepared from paraffin-embedded samples of E. tenella-infected chicken caeca (144 h p.i.) and T. gondii-infected cat intestine (day 7). Deparaffinisation, antigen retrieval and immunofluorescence analysis were carried out as described previously [17], using antibodies described in the Results section. Alternatively, unsporulated oocysts were vortexed with an equal volume of glass beads (710-1,180 $\mu \mathrm{m}$, Sigma) and air-dried onto glass slides prior to immunofluorescence assays. Alexa Fluor 488 goat anti-mouse (green) and Alexa Fluor 594 goat anti-rabbit (red) secondary antibodies were used at a dilution of 1 in 500 for detecting mouse and rabbit primary antibodies, respectively, and DAPI counterstaining was used at $1 \mu \mathrm{g} / \mathrm{ml}$. Samples were mounted in VECTASHIELD ${ }^{\circ}$ Mounting Medium (Vector Laboratories). Epifluorescence imaging was performed using a Leica DMI $6000 \mathrm{~B}$ microscope and the Leica LAS AF software. Confocal imaging was performed using a Leica SP2 AOBS confocal laser-scanning microscope and the Leica Confocal software for data collection, with subsequent deconvolution using the Huygens Essential (Scientific Volume Imaging B.V.) software.

\section{Western blot}

Protein samples were prepared from E. tenella and uninfected host caecal samples and analysed by Western blot under reducing and alkylating conditions as described previously [17]. Again, the initial lysis of oocyst samples was achieved by vortexing with glass beads (as described above). Protein concentration was determined using a NanoDrop ND-1000 spectrophotometer. Membranes were probed with either mouse or rabbit sera (at dilutions described in the results) and subsequently with goat anti-mouse or anti-rabbit IgG (heavy and light chain), horseradish peroxidase conjugates (Life technologies) diluted at 1 in 5,000. Probed membranes were developed using CPS-1 Chemiluminescent Peroxidase Substrate-1 (Sigma) according to manufacturer's instructions. The membranes were visualised using the VersDocTM Imaging System (BioRad) and images analysed using the Quantity One 1-D Analysis Software (BioRad).

\section{Gene annotation and sequences analysis}

Where possible, existing gene annotations available on www.toxodb.org were used for E. tenella genes described in this study. For hypothetical genes, additional annotation was achieved through Blast2Go analysis (www.blast2go.com) 
of the predicted protein sequences, using a cut-off of E-Value $<0.0005$. The identification of potential homologues of $E$. tenella proteins was also carried out using BlastP on the non-redundant NCBI database or on www.toxodb.org. Multiple alignments of protein and DNA sequences were performed using ClustalW (http://www.ebi. ac.uk/Tools/msa/clustalw2/). The prediction of signal peptides in protein sequences was carried out using the SignalP 4.1 Server (www.cbs.dtu.dk/services/SignalP/).

\section{Statistical analysis}

One-way analysis of variance (ANOVA) were carried out on qRT-PCR results using GraphPad Prism ${ }^{\circ}$ Version 6.03 (GraphPad Software Inc., USA).

\section{Ethics statement}

Animal experiments performed in Australia were carried out according to the specific regulations of the Australian Code of Practice for the Care and Use of Animals for Scientific Purposes. Mouse experiments performed in Cairns (permit number A1812) were approved by the Animal Ethics Committee at James Cook University. Chicken experiments performed in Sydney were approved by the University of Technology Animal Care and Ethics Committee (protocols 2008-096 and 2008-188). Animal experiments performed in Zurich, Switzerland were approved by the Veterinary Office and the Ethics Committee of the Canton of Zurich (Kantonales Veterinaramt Zurich, Obstgartenstrasse 21, 8090 Zurich, Switzerland) and carried out according to Swiss law and guidelines on Animal Welfare and the specific regulations of the Canton of Zurich. Permit number 171/2013 covers the production of polyclonal sera in mice, while permit number 108/2010 covers all mouse and cat experiments required for $T$. gondii sexual stage propagation. Experiments performed in Tours, France (protocol registration 2012-11-9) were approved by the ethics committee CEEA VdL and were carried out according to French legislation (French Government Decree 2001-464) and EEC regulations (86/609/CEE).

\section{Additional files}

Additional file 1: Sequencing the E. tenella gametocyte

transcriptome using RNA Seq. (a, b) A gametocyte sample purified from E. tenella-infected chickens at $144 \mathrm{~h}$ post-infection viewed under bright-field (a) or after DAPI staining (b). DAPI stains the nuclei of multiple microgametes and faintly stains the nuclei of a single macrogametocyte. Scale $=10 \mu \mathrm{m}$. (c) Total RNA extracted from triplicate samples of $E$. tenella gametocytes (Ga, Gb, Gc), merozoites (Ma, Mb, Mc) and sporozoites (Sa, Sb, Sc) was analysed using a Bioanalyzer 2100 (Agilent). Parasite large ribosomal RNA bands, $26 \mathrm{~S}$ and 18S, are detected in all samples, while a faint, host-specific $28 \mathrm{~S}$ ribosomal RNA band is detected in the gametocytes samples only (arrow). (d) The number of total paired reads generated for each of the nine RNA Seq experiments is plotted alongside the number of reads mapping uniquely to exon models of $E$. tenella.
Additional file 2: Total paired reads mapped uniquely to predicted exon models of $E$. tenella. Listed for each of the nine RNA Seq libraries are the total number of paired reads uniquely mapped to the predicted exon model of each of the 8,786 E. tenella genes. The nine RNA Seq libraries were produced from triplicate samples of $E$. tenella gametocytes (Ga, Gb, Gc), merozoites (Ma, Mb, Mc) and sporozoites (Sa, Sb, Sc).

Additional file 3: Differential expression of gene transcription in $E$. tenella gametocytes. Listed for each of the 8,786 E. tenella genes are the normalised counts calculated by DESeq in each of the three developmental stages (gametocytes, merozoites and sporozoites), along with the calculated differential expression in gametocytes over either merozoites or sporozoites. The fold change, log2 fold change, p-value and adjusted $p$-value are also listed. 'Max' indicates where transcript was exclusively detected in gametocytes, 'Min' indicates where transcript was exclusively detected in the reference asexual stage and ' $N A^{\prime}$ ' indicates where transcript was not detected in either stage.

Additional file 4: Transcript abundance and annotation of E. tenella upregulated gametocyte transcripts. Listed for each of the 863 upregulated E. tenella gametocyte transcripts is the publicly available gene annotation (www.toxodb.org), an annotation derived manually from Blast2Go analysis and the manually assigned biological function. As a measure of transcript abundance, the FPKM (Fragments Per Kilobase of exon model per Million mapped reads) of each gene is listed for each of the three developmental stages of E. tenella.

Additional file 5: Sequence alignment of ETH_00019840 and ETH_00019845 proteins. The amino acid sequences of ETH_00019840 and ETH_00019845, coded by the first and second most abundant upregulated $E$. tenella gametocyte transcripts (respectively), were aligned using ClustalW. '*' indicates the alignment of identical amino acids, while ':' and '.' indicate the alignment of conserved and partially conserved amino acids.

Additional file 6: Sequence alignment of ETH_00019835 and ETH_00032470 proteins. The amino acid sequences of ETH_00019835 and ETH_00032470, coded by the third and fourth most abundant upregulated E. tenella gametocyte transcripts, were aligned using ClustalW. ${ }^{\prime * \prime}$ indicates the alignment of identical amino acids, while ".' and '.' indicate the alignment of conserved and partially conserved amino acids.

Additional file 7: Conservation of cysteine-rich oocyst wall proteins. The amino acid sequences of EtOWP6 (ETH_00012470), TgOWP6 (TGME49_286250) and CpOWP6 (cgd4_3090) were aligned using ClustalW. The position of cysteine residues which mark the beginning of cysteine-rich Type I repeats are indicated with arrows. ${ }^{\prime * \prime}$ indicates the alignment of identical amino acids, while ':' and '.' indicate the alignment of conserved and partially conserved amino acids.

Additional file 8: Conservation of Hypothetical Oocyst Wall Protein 1 (HOWP1) in E. tenella and T. gondii. The amino acid sequences of EtHOWP1 (ETH_00018895) and TgHOWP1 (TGME49_316890) were aligned using ClustalW. ${ }^{* \prime}$ indicates the alignment of identical amino acids, while '?' and ". indicate the alignment of conserved and partially conserved amino acids.

Additional file 9 Conservation of Amine Oxidase 2 (AO2) in E. tenella and T. gondii. The amino acid sequences of (i) the MAM domain and (ii) the Cu + amine oxidase enzyme domain of EtAO2 (ETH_00028385) and TgAO2 (TGME49_086780) were aligned using ClustalW. The MAM domain corresponds to residues 235-408 and 176-347 of EtAO2 and TgAO2, respectively. The $\mathrm{Cu}+$ amine oxidase enzyme domain corresponds to residues 1,178-1,612 and 1,109-1,505 of EtAO2 and TgAO2, respectively. ${ }^{* \prime \prime}$ indicates the alignment of identical amino acids, while ".' and ".' indicate the alignment of conserved and partially conserved amino acids.

Additional file 10: Primers used in this study. The name, sequence and direction of each primer used throughout this study are listed. Also provided are the target gene name/annotation, template source for PCR and purpose of amplification. Additional comments are also provided.

\section{Abbreviations}

FPKM: Fragments per kilobase of exon model per million mapped reads; CDS: Coding sequence; DE: Differential expression; WFB: Wall forming bodies; HOWP1: Hypothetical oocyst wall protein 1; GCS1-HAP2: Generative cell specific 1-HAPLESS 2. 


\section{Competing interests}

The authors declare that they have no competing interests.

\section{Authors' contributions}

RAW participated in the overall design of the study, carried out a majority of the experiments and data analysis and drafted the manuscript. PAS performed, analysed and interpreted the experiments for the identification and characterisation of subtilisins and oxidoreductases. CMM helped plan, analyse and interpret the experiments for the identification and characterization of subtilisins and oxidoreductases. CL performed RNA quality assessment, coordinated the RNA Seq and prepared paraffin blocks for subsequent IFA experiments. MO converted raw RNA Seq data into normalized DESeq reads, ran the hierarchical clustering and produced DE tables. CR helped design and perform immunolocalisation experiments and contributed to RT-PCR analysis of ethap2 and sequence analysis. RME carried out some of the mouse immunisations and sera collection. FB prepared sporozoite RNA samples. PD participated in the coordination of mouse immunisation. $\mathrm{ABH}$ participated in the coordination of RNA Seq and data analysis. NCS conceived the study and helped draft the manuscript. All authors read and approved the final manuscript.

\section{Acknowledgements}

We are grateful to the staff at the Functional Genomics Centre Zurich for carrying out RNA Seq experiments and for providing ongoing technical support. We are grateful to the staff of the Ernst Facility at the University of Technology, Sydney, for their care of the chickens used in this study. NCS gratefully acknowledges the Australian Research Council for Discovery Project funding (DP0664013) and NCS and FB are grateful for an Australian Government Department of Innovation, Industry, Science and Research International Science Linkage grant (FR1 10089). NCS is supported by a grant from the Bellberry Foundation. RAW was supported by a Swiss Government Excellence Scholarship: Postdoctoral Scholarship from the Swiss Confederation. PAS was supported by an Australian Postgraduate Award Scholarship and an Australian Society for Parasitology Researcher Exchange, Training and Travel Award. None of the funding bodies played any role in: the design, collection, analysis, or interpretation of data; the writing of the manuscript; or the decision to submit the manuscript for publication. Finally, we would like to express our deep sadness for the loss of a wonderful researcher and friend, Fabien Brossier, who passed away on the 11th January 2014.

\section{Author details}

${ }^{1}$ Queensland Tropical Health Alliance Research Laboratory, Australian Institute of Tropical Health and Medicine, James Cook University, Cairns Campus, McGregor Road, Smithfield, QLD 4878, Australia. Institute of Parasitology, University of Zurich, Winterthurerstrasse 266a, CH-8057, Zürich, Switzerland. ${ }^{3}$ College of Public Health, Medical and Veterinary Sciences, James Cook University, Cairns Campus, McGregor Road, Smithfield, QLD 4878, Australia. ${ }^{4}$ Department of Farm Animal, University of Zurich, Winterthurerstrasse, CH-8057 Zürich, Switzerland. ${ }^{5}$ Functional Genomics Center Zurich, Winterthurerstrasse, $\mathrm{CH}-8057$ Zürich, Switzerland. ${ }^{6}$ Apicomplexes et Immunité Mucosale, INRA, UMR1282, Infectiologie et Santé Publique, F-37380 Nouzilly, France. ${ }^{7}$ Université François Rabelais de Tours, UMR1282, Infectiologie et Santé Publique, F-37000 Tours, France.

\section{Received: 18 September 2014 Accepted: 29 January 2015}

\section{Published online: 18 February 2015}

\section{References}

1. Shirley MW, Ivens A, Gruber A, Madeira AM, Wan KL, Dear PH, et al. The Eimeria genome projects: a sequence of events. Trends Parasitol. 2004;20:199-201.

2. Dalloul RA, Lillehoj HS. Poultry coccidiosis: recent advancements in control measures and vaccine development. Expert Rev Vaccines. 2006;5:143-63.

3. Shirley MW, Blake D, White SE, Sheriff R, Smith AL. Integrating genetics and genomics to identify new leads for the control of Eimeria spp. Parasitology. 2004;128 Suppl 1:S33-42.

4. Walker RA, Ferguson DJ, Miller CM, Smith NC. Sex and Eimeria: a molecular perspective. Parasitology. 2013;140:1701-17.

5. Wallach M. The development of CoxAbic a novel vaccine against vaccine. World Poultry. 2002;18:2-4.
6. Wallach MG, Ashash U, Michael A, Smith NC. Field application of a subunit vaccine against an enteric protozoan disease. PLoS One. 2008;3:e3948.

7. Novaes J, Rangel LT, Ferro M, Abe RY, Manha AP, de Mello JC, et al. A comparative transcriptome analysis reveals expression profiles conserved across three Eimeria spp. of domestic fowl and associated with multiple developmental stages. Int J Parasitol. 2012;42:39-48.

8. Matsubayashi M, Hatta T, Miyoshi T, Anisuzzaman, Sasai K, Shimura K, et al. High-throughput RNA sequencing profiles and transcriptional evidence of aerobic respiratory enzymes in sporulating oocysts and sporozoites of Eimeria tenella. Infect Genet Evol. 2013;18:269-76.

9. Amiruddin N, Lee XW, Blake DP, Suzuki Y, Tay YL, Lim LS, et al. Characterisation of full-length CDNA sequences provides insights into the Eimeria tenella transcriptome. BMC Genomics. 2012;13:21.

10. Radke JR, Behnke MS, Mackey AJ, Radke JB, Roos DS, White MW. The transcriptome of Toxoplasma gondii. BMC Biol. 2005;3:26.

11. Behnke MS, Wootton JC, Lehmann MM, Radke JB, Lucas O, Nawas J, et al. Coordinated progression through two subtranscriptomes underlies the tachyzoite cycle of Toxoplasma gondii. PLoS One. 2010;5:e12354.

12. Fritz HM, Buchholz KR, Chen X, Durbin-Johnson B, Rocke DM, Conrad PA, et al. Transcriptomic analysis of toxoplasma development reveals many novel functions and structures specific to sporozoites and oocysts. PLoS One. 2012;7:e29998.

13. Behnke MS, Zhang TP, Dubey JP, Sibley LD. Toxoplasma gondii merozoite gene expression analysis with comparison to the life cycle discloses a unique expression state during enteric development. BMC Genomics. 2014;15:350.

14. Le Roch KG, Zhou Y, Blair PL, Grainger M, Moch JK, Haynes JD, et al. Discovery of gene function by expression profiling of the malaria parasite life cycle. Science. 2003;301:1503-8.

15. Silvestrini F, Bozdech Z, Lanfrancotti A, Giulio ED, Bultrini E, Picci L, et al. Genome-wide identification of genes upregulated at the onset of gametocytogenesis in Plasmodium falciparum. Mol Biochem Parasitol. 2005;143:100-10.

16. Belli SI, Ferguson DJ, Katrib M, Slapetova I, Mai K, Slapeta J, et al. Conservation of proteins involved in oocyst wall formation in Eimeria maxima, Eimeria tenella and Eimeria acervulina. Int J Parasitol. 2009;39:1063-70.

17. Walker RA, Slapetova I, Slapeta J, Miller CM, Smith NC. The glycosylation pathway of Eimeria tenella is upregulated during gametocyte development and may play a role in oocyst wall formation. Eukaryot Cell. 2010;9:127-35.

18. Katrib M, Ikin RJ, Brossier F, Robinson M, Slapetova I, Sharman PA, et al. Stage-specific expression of protease genes in the apicomplexan parasite, Eimeria tenella. BMC Genomics. 2012;13:685.

19. Anders S, McCarthy DJ, Chen Y, Okoniewski M, Smyth GK, Huber W, et al. Count-based differential expression analysis of RNA sequencing data using R and Bioconductor. Nat Protoc. 2013;8:1765-86.

20. Miska KB, Fetterer RH, Lillehoj HS, Jenkins MC, Allen PC, Harper SB. Characterisation of macrophage migration inhibitory factor from Eimeria species infectious to chickens. Mol Biochem Parasitol. 2007;151:173-83.

21. Miska KB, Kim S, Fetterer RH, Dalloul RA, Jenkins MC. Macrophage migration inhibitory factor (MIF) of the protozoan parasite Eimeria influences the components of the immune system of its host, the chicken. Parasitol Res. 2013;112:1935-44.

22. Brothers VM, Kuhn I, Paul LS, Gabe JD, Andrews WH, Sias SR, et al. Characterization of a surface antigen of Eimeria tenella sporozoites and synthesis from a cloned cDNA in Escherichia coli. Mol Biochem Parasitol. 1988;28:235-47.

23. Wallach MG, Mencher D, Yarus S, Pillemer G, Halabi A, Pugatsch T. Eimeria maxima: identification of gametocyte protein antigens. Exp Parasitol. 1989;68:49-56.

24. Mouafo AN, Weck-Heimann A, Dubremetz JF, Entzeroth R. Monoclonal antibodies specific for the two types of wall-forming bodies of Eimeria tenella macrogametes (Coccidia, Apicomplexa). Parasitol Res. 2002;88:217-24.

25. Krucken J, Hosse RJ, Mouafo AN, Entzeroth R, Bierbaum S, Marinovski P, et al. Excystation of Eimeria tenella sporozoites impaired by antibody recognizing gametocyte/oocyst antigens GAM22 and GAM56. Eukaryot Cell. 2008;7:202-11.

26. Possenti A, Cherchi S, Bertuccini L, Pozio E, Dubey JP, Spano F. Molecular characterisation of a novel family of cysteine-rich proteins of Toxoplasma gondii and ultrastructural evidence of oocyst wall localisation. Int J Parasitol. 2010;40:1639-49. 
27. Templeton TJ, Lancto CA, Vigdorovich V, Liu C, London NR, Hadsall KZ, et al. The Cryptosporidium oocyst wall protein is a member of a multigene family and has a homolog in Toxoplasma. Infect Immun. 2004;72:980-7.

28. Spano F, Puri C, Ranucci L, Putignani L, Crisanti A. Cloning of the entire COWP gene of Cryptosporidium parvum and ultrastructural localization of the protein during sexual parasite development. Parasitology. 1997;114(Pt 5):427-37.

29. Flowers SA. The proteome of the coccidian oocyst wall. University of Technology, Sydney, Faculty of Science; 2011.

30. Belli SI, Wallach MG, Luxford C, Davies MJ, Smith NC. Roles of tyrosine-rich precursor glycoproteins and dityrosine- and 3,4-dihydroxyphenylalaninemediated protein cross-linking in development of the oocyst wall in the coccidian parasite Eimeria maxima. Eukaryot Cell. 2003;2:456-64.

31. Ferguson DJ, Belli SI, Smith NC, Wallach MG. The development of the macrogamete and oocyst wall in Eimeria maxima: immuno-light and electron microscopy. Int J Parasitol. 2003;33:1329-40.

32. Cowper B, Matthews S, Tomley F. The molecular basis for the distinct host and tissue tropisms of coccidian parasites. Mol Biochem Parasitol. 2012;186:1-10.

33. Mai K, Smith NC, Feng ZP, Katrib M, Slapeta J, Slapetova I, et al. Peroxidase catalysed cross-linking of an intrinsically unstructured protein via dityrosine bonds in the oocyst wall of the apicomplexan parasite, Eimeria maxima. Int J Parasitol. 2011;41:1157-64.

34. Beckmann G, Bork P. An adhesive domain detected in functionally diverse receptors. Trends Biochem Sci. 1993;18:40-1.

35. Belli SI, Lee M, Thebo P, Wallach MG, Schwartsburd B, Smith NC. Biochemical characterisation of the 56 and $82 \mathrm{kDa}$ immunodominant gametocyte antigens from Eimeria maxima. Int J Parasitol. 2002;32:805-16.

36. Bushkin GG, Motari E, Magnelli P, Gubbels MJ, Dubey JP, Miska KB, et al. beta-1,3-Glucan, Which Can Be Targeted by Drugs, Forms a Trabecular Scaffold in the Oocyst Walls of Toxoplasma and Eimeria. MBio. 2012:3:e00258-12.

37. Bushkin GG, Motari E, Carpentieri A, Dubey JP, Costello CE, Robbins PW, et al. Evidence for a structural role for acid-fast lipids in oocyst walls of Cryptosporidium, Toxoplasma, and Eimeria. MBio. 2013;4:e00387-00313.

38. Khan SM, Franke-Fayard B, Mair GR, Lasonder E, Janse CJ, Mann M, et al. Proteome analysis of separated male and female gametocytes reveals novel sex-specific Plasmodium biology. Cell. 2005;121:675-87.

39. Wass MN, Stanway R, Blagborough AM, Lal K, Prieto JH, Raine D, et al. Proteomic analysis of Plasmodium in the mosquito: progress and pitfalls. Parasitology. 2012;139:1131-45.

40. Straschil U, Talman AM, Ferguson DJ, Bunting KA, Xu Z, Bailes E, et al. The Armadillo repeat protein PF16 is essential for flagellar structure and function in Plasmodium male gametes. PLoS One. 2010;5:e12901.

41. Farr H, Gull K. Functional studies of an evolutionarily conserved, cytochrome b5 domain protein reveal a specific role in axonemal organisation and the general phenomenon of post-division axonemal growth in trypanosomes. Cell Motil Cytoskeleton. 2009;66:24-35.

42. Balhorn R. The protamine family of sperm nuclear proteins. Genome Biol 2007:8:227.

43. Ohsako S, Hayashi Y, Bunick D. Molecular cloning and sequencing of calnexin-t. An abundant male germ cell-specific calcium-binding protein of the endoplasmic reticulum. J Biol Chem. 1994;269:14140-8.

44. Ikawa M, Wada I, Kominami K, Watanabe D, Toshimori K, Nishimune Y, et al. The putative chaperone calmegin is required for sperm fertility. Nature. 1997;387:607-11.

45. Yukitake H, Furusawa M, Taira T, Iguchi-Ariga SM, Ariga H. AAT-1, a novel testis-specific AMY-1-binding protein, forms a quaternary complex with AMY-1, A-kinase anchor protein 84, and a regulatory subunit of CAMP-dependent protein kinase and is phosphorylated by its kinase. J Biol Chem. 2002;277:45480-92.

46. Sutton KA, Jungnickel MK, Wang Y, Cullen K, Lambert S, Florman HM. Enkurin is a novel calmodulin and TRPC channel binding protein in sperm. Dev Biol. 2004;274:426-35.

47. Wong JL, Johnson MA. Is HAP2-GCS1 an ancestral gamete fusogen? Trends Cell Biol. 2010:20:134-41.

48. Liu Y, Tewari R, Ning J, Blagborough AM, Garbom S, Pei J, et al. The conserved plant sterility gene HAP2 functions after attachment of fusogenic membranes in Chlamydomonas and Plasmodium gametes. Genes Dev. 2008;22:1051-68.
49. Blagborough AM, Sinden RE. Plasmodium berghei HAP2 induces strong malaria transmission-blocking immunity in vivo and in vitro. Vaccine. 2009;27:5187-94.

50. Miura K, Takashima E, Deng B, Tullo G, Diouf A, Moretz SE, et al. Functional comparison of Plasmodium falciparum transmission-blocking vaccine candidates by the standard membrane-feeding assay. Infect Immun. 2013;81:4377-82.

51. Wong JL, Leydon AR, Johnson MA. HAP2(GCS1)-dependent gamete fusion requires a positively charged carboxy-terminal domain. PLoS Genet. 2010;6:e1000882.

52. Sprunck S, Rademacher S, Vogler F, Gheyselinck J, Grossniklaus U, Dresselhaus T. Egg cell-secreted EC1 triggers sperm cell activation during double fertilization. Science. 2012;338:1093-7.

53. Laxer MA, Healey MC, Youssef NN. Production of monoclonal antibodies specific for Eimeria tenella microgametocytes. J Parasitol. 1987;73:611-6.

54. Long PL. Observations on the oocyst production and viability of Eimeria mivati and E. tenella in the chorioallantois of chicken embryos incubated at different temperatures. Z Parasitenkd. 1972;39:27-37.

55. Fritz HM, Bowyer PW, Bogyo M, Conrad PA, Boothroyd JC. Proteomic analysis of fractionated Toxoplasma oocysts reveals clues to their environmental resistance. PLoS One. 2012;7:e29955.

56. Shirley MW. Eimeria species and strains of chickens. In: Eckert J, Braun R, Shirley MW, Coudert P, editors. Biotechnology: Guidelines on Techniques in Coccidiosis Research. Luxembourg: European Commission; 1995. p. 1-24.

57. Shirley MW. Development of a live attenuated vaccine against coccidiosis of poultry. Parasite Immunol. 1989;11:117-24.

58. Rieux A, Gras S, Lecaille F, Niepceron A, Katrib M, Smith NC, et al. Eimeripain, a cathepsin B-like cysteine protease, expressed throughout sporulation of the apicomplexan parasite Eimeria tenella. PLoS One. 2012;7:e31914.

59. Reid AJ, Blake DP, Ansari HR, Billington K, Browne HP, Bryant JM, et al. Genomic analysis of the causative agents of coccidiosis in domestic chickens. Genome Res. 2014;24:1676-85.

60. Kim D, Pertea G, Trapnell C, Pimentel H, Kelley R, Salzberg SL. TopHat2: accurate alignment of transcriptomes in the presence of insertions, deletions and gene fusions. Genome Biol. 2013;14:R36.

61. Anders S, Huber W. Differential expression analysis for sequence count data. Genome Biol. 2010;11:R106.

62. Studier FW. Protein production by auto-induction in high density shaking cultures. Protein Expr Purif. 2005;41:207-34.

\section{Submit your next manuscript to BioMed Central and take full advantage of:}

- Convenient online submission

- Thorough peer review

- No space constraints or color figure charges

- Immediate publication on acceptance

- Inclusion in PubMed, CAS, Scopus and Google Scholar

- Research which is freely available for redistribution 